\title{
The sedentary survey of extreme high energy peaked BL Lacs
}

\section{The catalog and spectral properties ${ }^{\star} \star \star \star$}

\author{
P. Giommi ${ }^{1}$, S. Piranomonte ${ }^{1}$, M. Perri ${ }^{1}$, and P. Padovani ${ }^{2,3, \star \star \star}$ \\ 1 ASI Science Data Center, ASDC, Agenzia Spaziale Italiana c/o ESRIN, via G. Galilei, 00044 Frascati, Italy \\ e-mail: paolo.giommi@asi.it \\ 2 Space Telescope Science Institute, 3700 San Martin Drive, Baltimore, MD 21218, USA \\ 3 Affiliated to the Space Telescope Division of the European Space Agency, ESTEC, Noordwijk, The Netherlands
}

Received 4 August 2004 / Accepted 29 November 2004

\begin{abstract}
The multi-frequency "Sedentary Survey" is a deep, statistically complete, radio flux limited sample comprising 150 BL Lacertae objects distinguished by their extremely high X-ray to radio flux ratio $\left(f_{\mathrm{x}} / f_{\mathrm{r}}\right)$, ranging from five hundred to over five thousand times that of typical BL Lacs discovered in radio surveys. This large excess of high energy photons compared to radio emission is thought to be due to synchrotron radiation that in these sources reaches the UV or the X-ray band. The name "Sedentary Survey" originates from the multi-frequency technique used to select the sample that was expected to be so efficient as to allow the conduction of some preliminary statistical studies even without the need to identify the candidates through optical spectroscopy. The details of the selection criteria and the preliminary results have been published in Giommi et al. (1999, MNRAS, 310, 465). In this paper we present the final, 100\% identified, catalog together with the optical, X-ray and broad-band Spectral Energy Distributions (SED) constructed combining literature multi-frequency data with non-simultaneous optical observations and BeppoSAX X-ray data, when available. The SEDs confirm that the peak of the synchrotron power in these objects is located at very high energies. BeppoSAX wide band X-ray observations show that, in most cases, the X-ray spectra are convex and well described by a logarithmic parabola model peaking (in a $v f(v)$ vs. $v$ representation) between 0.02 to several keV. Although detailed X-ray spectral data are available for only about one fifth of the sources the observed peaks never reach energies well above $10 \mathrm{keV}$ (as in Mkn 501 during the large X-ray flare of April 1997 and in 1ES 2344+514 in December 1996) implying that hard X-ray synchrotron peak energies are rare and probably associated with strong flaring events.

Owing to the high synchrotron energies involved most of the sources in the catalog are likely to be TeV emitters, with the closest and brightest ones probably detectable by the present generation of Cherenkov telescopes. However, only 50\% (3 out of 6) of the presently established TeV BL Lacs are actually included in the survey suggesting that the hardest peaks may be associated with secondary synchrotron components that can be detected only above the soft X-ray band. The existence of secondary emission regions is suggested by the strong X-ray spectral curvature that in some objects predicts an optical flux much below the observed emission.

The optical spectrum of about one fourth of the sources is totally featureless hampering any redshift or luminosity determination. Because this implies that the non-thermal nuclear emission must be well above that of the host galaxy, these objects are likely to be the most powerful sources in the survey and therefore be examples of the yet unreported high radio luminosity-high energy peaked BL Lacs. The existence of such objects would be at odds with the claimed inverse proportionality between radio power and synchrotron peak energy known as the "blazar sequence".

At the low-power end of the luminosity dynamical range, where the non-thermal optical continuum falls below the emission from the host galaxy, recognition issues start becoming important since BL Lacs in this luminosity regime can hardly be recognized as such, but rather as radio galaxies or simply as elliptical galaxies. We have found a small sample of bright nearby elliptical galaxies that are candidate low radio power high energy peaked BL Lacs.
\end{abstract}

Key words. galaxies: BL Lacertae objects: general - X-rays: galaxies - galaxies: quasars: general - surveys

\footnotetext{
* Figures 10-34 are only available in electronic form at http://www . edpsciences.org

$\star \star$ Tables 1-3 are only available in electronic form at the CDS via anonymous ftp to cdsarc.u-strasbg.fr $(130.79 .128 .5)$ or via http://cdsweb.u-strasbg.fr/cgi-bin/qcat?]/A+A/434/385 $\star \star \star$ Current address: ST-ECF, European Southern Observatory, Karl-Schwarzschild-Str. 2, 85748 Garching bei München, Germany.
}

\section{Introduction}

BL Lacertae objects are a rare and very peculiar type of Active Galactic Nuclei (AGN). Their observational properties, which include super-luminal motion, strong and rapidly variable nonthermal radiation across the entire electromagnetic spectrum and a high degree of polarization, are believed to be the 
signature of strongly amplified radiation emitted in a relativistic jet closely aligned to the line of sight (e.g. Urry \& Padovani 1995).

These unusual physical and geometrical properties, combined with the peculiar cosmological evolution that distinguishes BL Lacs from other types of AGN, have made this class of sources the subject of intense research activity and of large multi-frequency observation campaigns.

Despite the fact that BL Lacs emit strongly over the entire electromagnetic spectrum, nearly all of presently known sources of this type have been discovered at radio or at $\mathrm{X}$-ray frequencies, or through a combination of these two bands. However, in some still poorly explored observing windows, like the millimeter/microwave region and the gammaray/TeV bands, BL Lacs, together with Flat Spectrum Radio Quasars (FSRQ), are expected to be one of the major constituents of the extra-galactic discrete source population. New large samples of these objects will certainly be built when deep surveys based on data from the Planck and GLAST space missions will become available in a few years.

The observed non-thermal emission in BL Lacs is thought to be due to synchrotron emission peaking (in a $\log (v f(v)-$ $\log (v)$ representation) between the far infrared and the hard $\mathrm{X}$-ray band, followed by Inverse Compton scattering up to very high energies. Those BL Lacs where the synchrotron peak is located at low energy (known as Low energy peaked BL Lacs, LBL, Padovani \& Giommi 1995) so far have been discovered mostly in radio surveys, while those where the synchrotron power reaches the UV or the X-ray band (High energy peaked BL Lacs, or HBL) have been discovered much more frequently in X-ray surveys.

The Sedentary Multi-frequency Survey (Giommi et al. 1999, hereafter referred to as Paper I) was designed to assemble a large and statistically well defined sample of HBL BL Lacs by exploiting the fact that the electromagnetic emission of these sources is so extreme that no other type of extra-galactic source type is known to possess a similar Spectral Energy Distribution (SED). By imposing radio, optical and X-ray flux ratios that are only consistent with the unique SEDs of HBL BL Lacs it is then possible to build large samples of these rare objects with very high selection efficiency.

The sample presented in Paper I included 155 BL Lac candidates, only $40 \%$ of which were at the time spectroscopically identified. However, it was estimated that the multi-frequency selection technique applied ensured that at least $85 \%$ of the candidates were genuine BL Lacs. That allowed the authors to derive, though in a preliminary way, some important statistical properties of HBL BL Lacs, such as their radio $\log N-\log S$ and Cosmological evolution. For that reason the survey was named "Sedentary".

The estimation of some of the fundamental properties of the sample, however, require the knowledge of luminosity, hence redshift which makes an identification campaign clearly necessary. This need prompted the organization of a dedicated optical spectroscopy observation program (see Piranomonte et al. 2004, hereafter Paper III) that, together with data collected by other independent groups, mostly aimed at the systematic identification of bright high Galactic latitude RASS sources
(Schwope et al. 2000; Bauer et al. 2000; Beckmann 2000; Anderson et al. 2003), led to the identification of all the candidates in the sample.

In this paper we present the complete cleaned sample, which now includes 150 objects following i) the removal of those candidates that the spectroscopic identification campaign did not confirm to be BL Lacs, and ii) the addition of 7 new BL Lacs that satisfy all criteria for inclusion in the survey but were not in the original sample because their $\alpha_{\text {ro }}$ was just below the threshold of 0.2 due to the optical contamination from the host galaxy which was not taken into account.

We also present a detailed spectral analysis based on broad band BeppoSAX archival data and the radio to X-ray SED of a selection of objects built using multi-frequency literature data, on our own optical observations and BeppoSAX data, when available.

The radio $\log N-\log S$, luminosity function and cosmological evolution have been presented in preliminary form in Paper I and in Perri et al. (2002), the final results are presented in a dedicated paper (Giommi et al. 2005, hereafter Paper IV).

\section{The sample}

A complete description of the Sedentary Survey sample is given in Paper I, in this section we summarize the main selection criteria and we refer the reader to the original paper for more details.

The sample was extracted from a large set of radio and $\mathrm{X}$-ray emitting sources selected through a cross-correlation between the RASS catalog of bright X-ray sources (Voges et al. $1999)$ and the NVSS catalog of radio $(1.4 \mathrm{GHz}$, Condon et al. 1998) sources. The following conditions were imposed to avoid the complications due to the Galactic plane and ensure that the sample is statistically complete above the radio flux limit of $f_{\mathrm{r}}=3.5 \mathrm{mJy}$

1. $|b|>20^{\circ}$;

2. $f_{\mathrm{x}} / f_{\mathrm{r}} \geq 3 \times 10^{-10} \mathrm{erg} \mathrm{cm}^{-2} \mathrm{~s}^{-1} \mathrm{Jy}^{-1}$;

3. $\alpha_{\mathrm{ro}}>0.2$;

4. $f_{\mathrm{r}} \geq 3.5 \mathrm{mJy}$;

5. RASSBSC count rate $\geq 0.1 \mathrm{cts} / \mathrm{s}$;

6. $V \leq 21$

where $\alpha_{\text {ro }}$ is the usual broad band spectral index between the radio $(5 \mathrm{GHz})$ and optical $(5000 \AA)$ fluxes and $V$ is the visual apparent magnitude of the optical counterpart.

Condition 1) limits the survey area to high Galactic latitude regions where soft X-ray absorption due to Galactic $N_{\mathrm{H}}$ is low; condition 2) imposes a very large $f_{\mathrm{x}} / f_{\mathrm{r}}$ flux ratio that can be reached by HBL BL Lacs only; condition 3) removes from the sample radio quiet sources, such as nearby Seyfert galaxies where the unrelated radio and X-ray flux may accidentally satisfy condition 2); conditions 4), 5) and 6) are necessary to ensure statistical completeness above $f_{\mathrm{r}} \geq 3.5 \mathrm{mJy}$.

\section{The catalog}

The fully identified complete sample including 150 extreme HBL BL Lacs is presented in Table 1 where Col. 1 gives the 


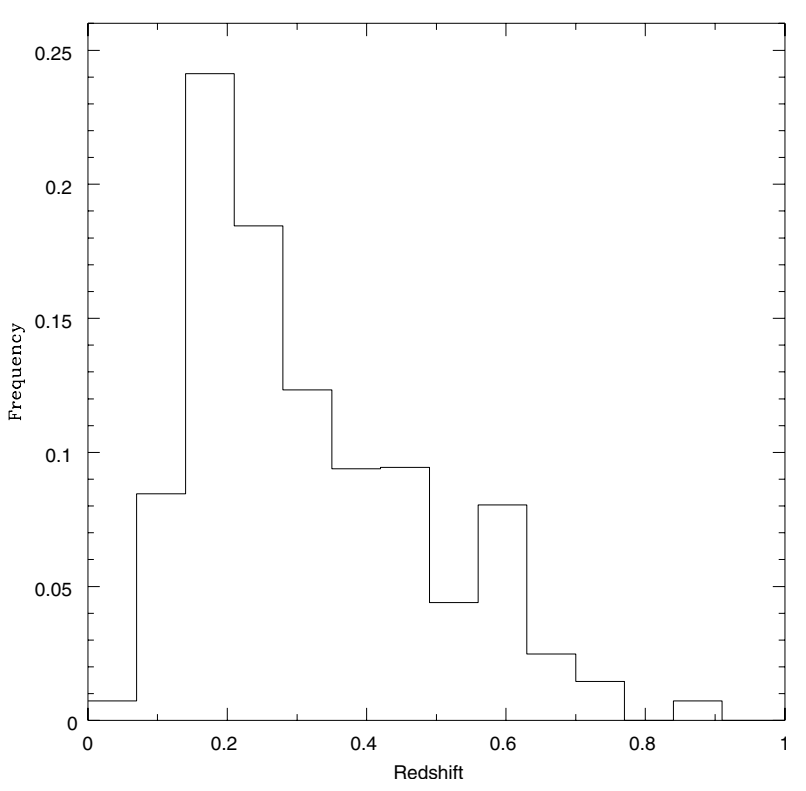

Fig. 1. The redshift distribution of the subsample of 111 BL Lacs with a measured redshift after de-convolving for the X-ray sky coverage of the survey (see also Landt et al. 2001).

source name built with the catalog identification code SHBL (where S stands for "Sedentary" survey and HBL for High energy peaked BL Lacs, Padovani \& Giommi 1995) and the arcsecond precision optical coordinates of the source taken from the APM (Irwin et al. 1994) and COSMOS (Yentis et al. 1992) on-line services; Col. 2 gives the RASS name; Cols. 3-5 give the X-ray flux $(0.1-2.4 \mathrm{keV})$, the radio flux $(20 \mathrm{~cm}$, from the NVSS survey), and the optical apparent $V$ magnitude (from APM and COSMOS, see Paper I) respectively; Col. 6 gives the redshift, when available; Col. 7 gives the reference for the optical identification.

The catalog is also available on the web at the following address http://www . asdc.asi.it/sedentary/ where additional data, including the broad band spectral energy distributions, finding charts and optical spectra (from Paper III) are also provided, when available.

Redshifts were measured for $111 \mathrm{BL}$ Lacs, mostly from spectral features due to the host galaxy. The other 39 sources ( $\sim 25 \%$ of the sample) remain without redshift since their optical spectrum does not show any emission or absorption lines.

The sample is flux limited, complete (that is all the sources above the flux limit are included) and 100\% identified and is therefore suitable for an unbiased investigation of the statistical properties of the population that it represents.

The main cosmological properties, such as radio $\log N-$ $\log S$, luminosity function and cosmological evolution, are studied in detail in Paper IV; in the following we provide only some basic statistics of the parameters listed in Table 1. The redshift and $V$ distributions, corrected for the X-ray sky coverage of the survey, are shown in Figs. 1 and 2 respectively. The average redshift of the subsample of 111 objects for which a value could be measured is $\langle z\rangle=0.32$ and is rather low compared to other radio loud $\mathrm{AGN}$ of similar or even higher radio flux (e.g. Padovani et al. 2003), but consistent with

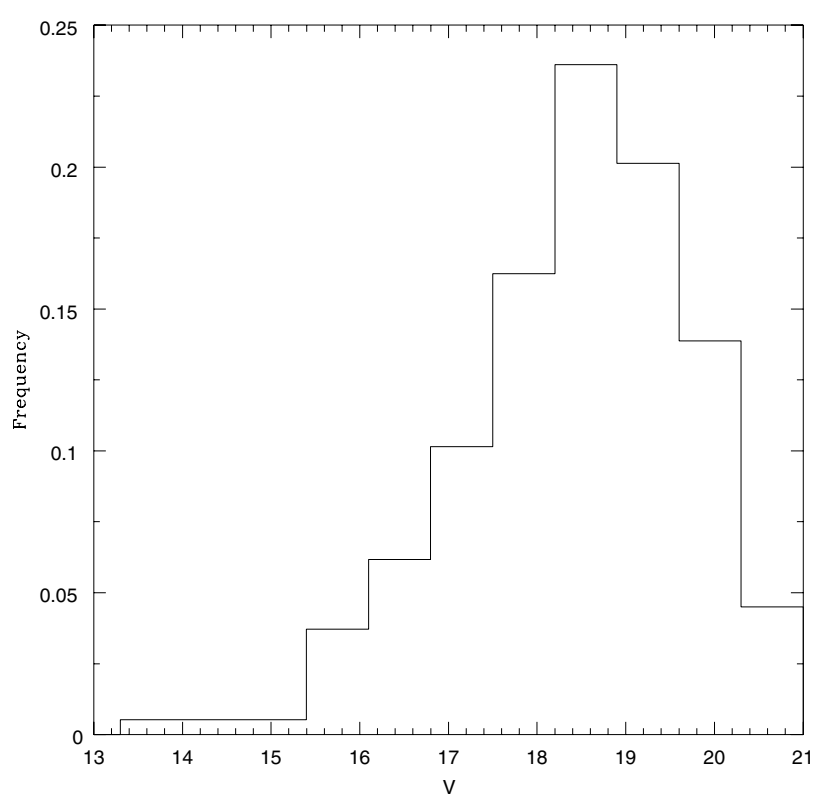

Fig. 2. The sky coverage corrected distribution of optical apparent magnitudes $(V)$ of all sources in the Sedentary survey. Note that the fraction of sources with $V \geq 20$ is very low suggesting that the condition 6 in the definition of the sample $(V \leq 21)$ only excluded a tiny fraction of sources.

earlier results about X-ray selected BL Lacs that have demonstrated the peculiar cosmological evolution of these sources (Bade et al. 1998a; Rector et al. 2000). However, the exclusion of the 39 sources without a redshift determination, which may well be distant, high luminosity objects (see below) implies that this value is likely to be only a lower limit.

The distribution of optical magnitudes (see Fig. 2) is sharply peaked around the mean value of $\langle V\rangle=18.4$ and only a few sources are fainter than $V \approx 20$. Condition number 6 in the sample definition criteria (i.e. $V \leq 21$ ) should therefore exclude a very small fraction of BL Lacs ( $\ 1-2 \%$, see also Paper I).

\subsection{Notes on individual objects}

\subsubsection{SHBL J040128.0+815312}

This source has been identified as an early-type galaxy by Bauer et al. 2000 but its X-ray and radio luminosities are rather high (about $5 \times 10^{44} \mathrm{erg} / \mathrm{s}$ and $3 \times 10^{31} \mathrm{erg} / \mathrm{s} / \mathrm{Hz}$ respectively). It is therefore likely that this source is a AGN, in the following we will assume that it is a BL Lac.

\subsubsection{SHBL J114535.1-034001, SHBL J235023.2-243603}

These X-ray/radio sources are within clusters of galaxies. However, since i) the RASS X-ray emission in these sources is not extended; ii) the radio emission coincides with a galaxy; and iii) from our optical spectroscopic campaign we have found that the $\mathrm{Ca} H \& \mathrm{~K}$ break in their optical spectrum is diluted by non-thermal radiation. We assume that these objects are BL Lacs in clusters. 

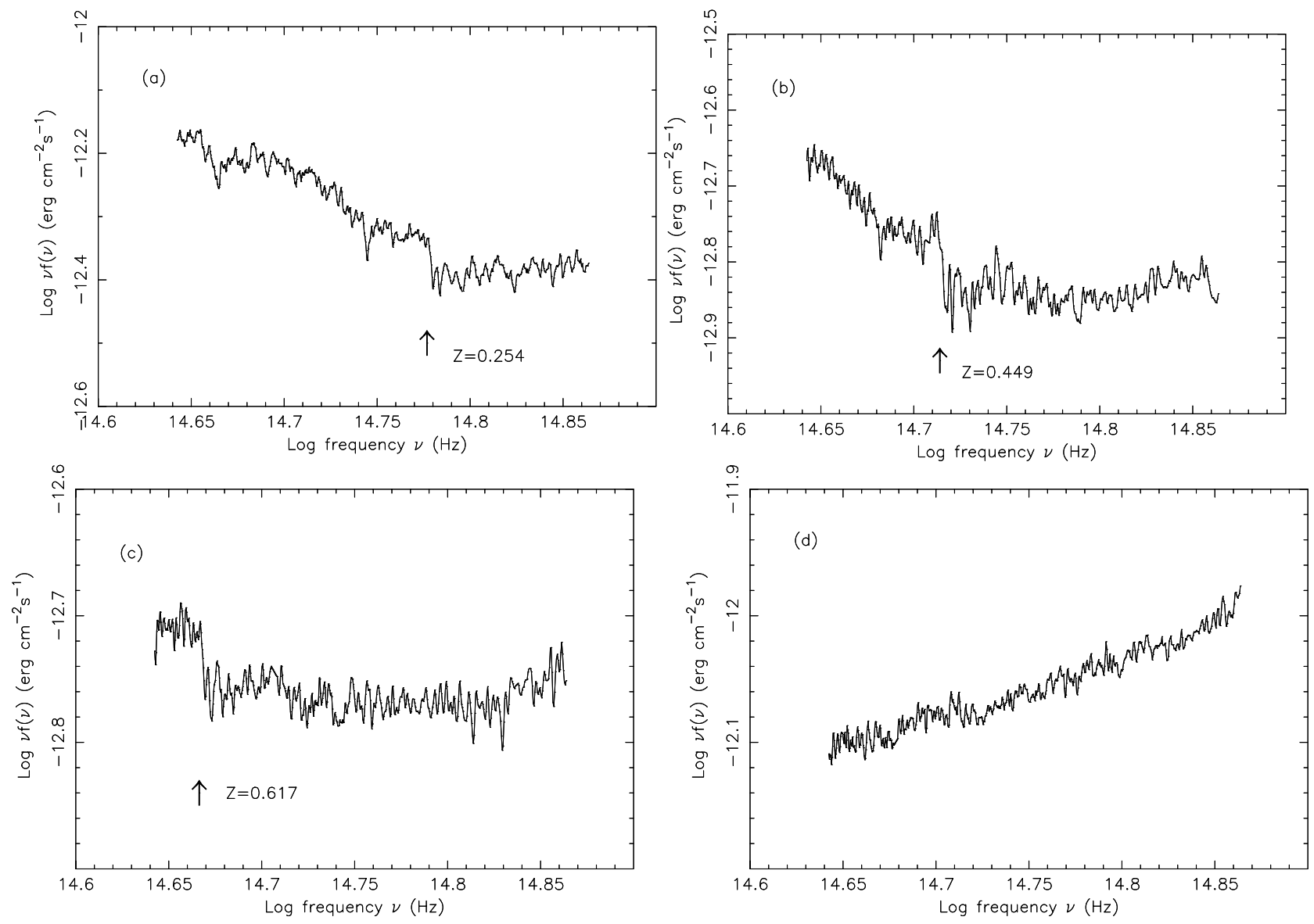

Fig. 3. SED of the optical region of the four BL Lac objects SHBLJ 104651.4-253545 a), SHBLJ 044230.1-001830 b), SHBLJ 020412.8-333342 c) and SHBLJ 150340.6-154113 d). Note that the optical output from the host galaxy (steep component) dominates the spectra of the sources a), b) and c) at frequencies below the Ca H\&K break (indicated by the arrow) while the nuclear non-thermal emission (flat component) is apparent at frequencies above the Ca H\&K break. The optical spectrum of source d) is instead totally dominated by its flat non-thermal component; no spectral features can be seen and therefore its redshift cannot be determined.

\section{Optical spectroscopy}

In Paper I only about $40 \%$ of the sources in the sample were confirmed BL Lacs. Although several other candidates were subsequently identified in a number of projects dedicated to the optical follow up observations of bright Rosat X-ray sources (Schwope et al. 2000; Bauer et al. 2000; Beckmann 2000), many objects would have remained unidentified without a dedicated optical spectroscopy program. We have therefore carried out an extensive optical identification campaign using the Kitt Peak National Observatory $4 \mathrm{~m}$ telescope, the ESO $3.6 \mathrm{~m}$ telescope at La Silla and the TNG $3.6 \mathrm{~m}$ telescope at La Palma, which allowed us to identify all sources of the survey. The results are described in detail in Paper III; in the following we summarize the main results.

Good quality optical spectra were obtained for the 76 objects which were either previously unidentified (58) or had been reported in the literature as BL Lacs (18) but no redshift was given and no information about the quality of the optical spectrum was reported (see Table 1). Out of the 58 unclassified candidates 50 were confirmed to be BL Lacs and 8 sources turned out to be emission line AGN.
The full set of spectra are reported and discussed in Paper III. In this paper we show the optical spectra of four representative sources located at different redshifts, (see Fig. 3) plotted in $\log (v f(v))$ vs. $\log (v)$ space which is normally used to plot broad band Spectral Energy Distributions (SED). We have chosen this graphical representation because it is particularly effective in enhancing broad features and changes in the spectral shape.

The data have been de-reddened and converted to units suitable for $v f(v)$ vs. $v$ SED plots with the IRAF $^{1}$ packages noao.onedspec.deredden and noao.onedspec.splot.

Figure 3 illustrates how the emitted power at optical frequencies is a blend between the optical output from the host galaxy (steep component), which dominates the spectrum at frequencies below the $\mathrm{Ca} H \& \mathrm{~K}$ break, and the non-thermal nuclear emission (flat component), that usually appears at frequencies above the $\mathrm{Ca} H \& \mathrm{~K}$ break. The clear trend in the balance between the non-thermal and the galaxian component with redshift strongly indicates that high redshift (which

\footnotetext{
${ }^{1}$ IRAF is distributed by the National Optical Astronomy Observatories, which are operated by the Association of the Universities for Research in Astronomy, Inc., under cooperative agreement with the National Science Foundation.
} 


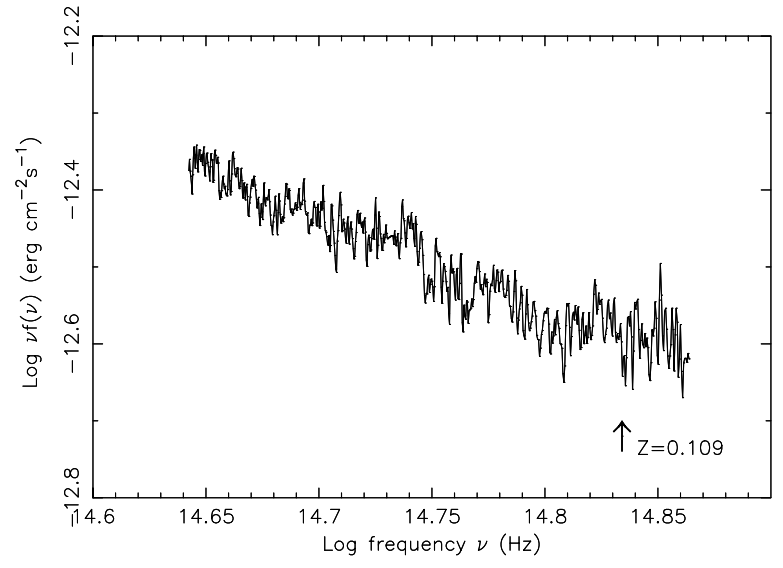

Fig. 4. SED of the optical region of SHBLJ 044127.4+150456. Most of the emission from this nearby $(z=0.109)$ object is red i.e. steep and dominated by the optical output from the host galaxy. The nuclear non-thermal emission (flat component) is barely apparent at frequencies above the $\mathrm{Ca} \mathrm{H} \& \mathrm{~K}$ break (indicated by the arrow).

on average implies high luminosity in a flux limited sample) sources are characterized by a flat, featureless optical spectrum. In sources located at redshifts higher than 0.7-0.8 the $\mathrm{Ca} H \& \mathrm{~K}$ break falls outside the optical band and the nonthermal emission, which at these distances and radio flux above the survey limit is much brighter than the typical host galaxy, completely dominates the optical spectrum. This calls for a high redshift location of the 39 sources in the sample whose optical spectrum is flat and completely featureless (see discussion in Sect. 4.2). An estimate of a lower limit to the redshift of these sources will be given in Papers III and IV.

\subsection{Elliptical galaxies or low luminosity BL Lacs?}

Since the luminosity of BL Lac host galaxies (giant ellipticals) is approximately constant (Wurtz et al. 1997; Urry et al. 2000) the blend between the non-thermal nuclear emission and the optical flux from the host galaxy must be a strong function of the luminosity of the BL Lac. This implies that at the low and high luminosity ends of the radio luminosity function the appearance of the optical spectrum of BL Lacs must be very different, as discussed in the previous paragraph and shown in Fig. 3 (see also Landt et al. 2002). In particular, the optical emission of low redshift-low luminosity BL Lacs, must be almost completely dominated by the emission from the host galaxy. An example of this effect is shown in Fig. 4 where the non-thermal component of SHBL J044127.4+150456, a $z=0.109 \mathrm{BL}$ Lac, is barely detectable only at frequencies above the $\mathrm{Ca} H \& \mathrm{~K}$ break. At even lower redshifts $(z \lesssim 0.1)$ the host galaxy totally dominates the spectrum and distinguishing between normal radio galaxies and BL Lacs becomes very difficult.

In this luminosity regime the $\mathrm{X}$-ray to radio flux ratio $\left(f_{\mathrm{x}} / f_{\mathrm{r}}\right)$ remains unaffected, but $\alpha_{\mathrm{ro}}$ is heavily contaminated by starlight and could decrease significantly pushing these low luminosity BL Lacs over the borderline between the HBL zone and the radio quiet zone $\left(\alpha_{\text {ro }}<0.2\right)$ thus biasing the sample at low luminosities. One object of this type, the elliptical galaxy

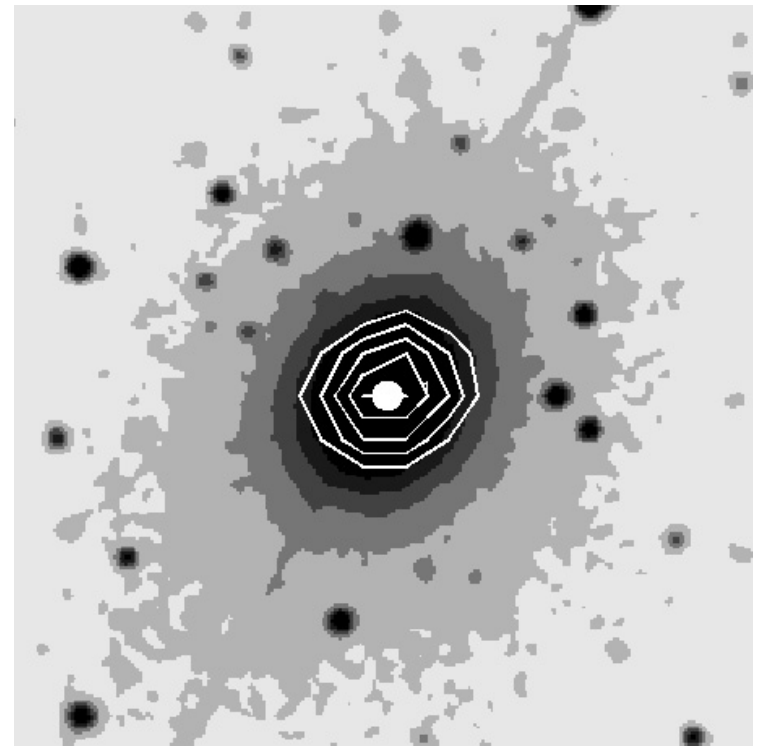

Fig. 5. The optical image and the radio iso-intensity contours of SHBL J020014.8+312545 = NGC 0777, a nearby elliptical galaxy and candidate HBL in the survey. The NVSS position (filled symbol) of the flat spectrum radio source is consistent with that of nucleus of NGC 0777.

IC 1459, was recently associated with a BL Lac by Giommi et al. (2002c).

We have been looking for similar bright elliptical galaxies/low luminosity HBLs in the original sample of high $f_{\mathrm{x}} / f_{\mathrm{r}}$ sources of Paper $\mathrm{I}\left(f_{\mathrm{x}} / f_{\mathrm{r}} \geq 3 \times 10^{-10} \mathrm{erg} \mathrm{cm}^{-2} \mathrm{~s}^{-1} \mathrm{Jy}^{-1}\right)$ among the objects that were excluded because their $\alpha_{\text {ro }}$ was below the threshold value of 0.2 . We have found the five objects that are reported in Table 2 where Cols. 1-3 give the SHBL, the RASS and other names; Col. 4 gives the redshift, when available; Col. 5 is the visual apparent magnitude; Col. 6 is the X-ray luminosity in the Rosat band and Col. 7 gives the source classification from NED. As an example Fig. 5 shows the optical image, taken from the ESO on-line service, of one of the objects in Table 2 (1RXS J020014.5+31254= NGC 0777) with the radio iso-intensity contours and the precise position from the NVSS survey overlayed. The flat/inverted radio spectrum $\left(\alpha_{1.4-2.38 \mathrm{GHz}}=-0.37 \pm 0.4, F(v) \propto v^{-\alpha}\right)$ coincident with the nucleus of the galaxy and the $f_{\mathrm{x}} / f_{\mathrm{r}}$ flux ratio make this source consistent with a high energy peaked BL Lac.

\subsection{High luminosity HBLs and the problem of redshift estimation}

At the bright end of the BL Lac radio luminosity function, especially for the case of HBL objects where, for the same radio luminosity the optical output is much higher than in LBL objects (see Fig. 6), the optical flux is totally dominated by the nonthermal, featureless nuclear emission which makes any redshift estimation a very difficult task. Figure 3 (panel d) is an example of a featureless optical spectrum belonging to a source that could be a high luminosity HBL. Since there are 39 such objects in the sample the fraction of high redshift $(z \gtrsim 0.8)-$ high luminosity objects may be as high as $\sim 25 \%$. 


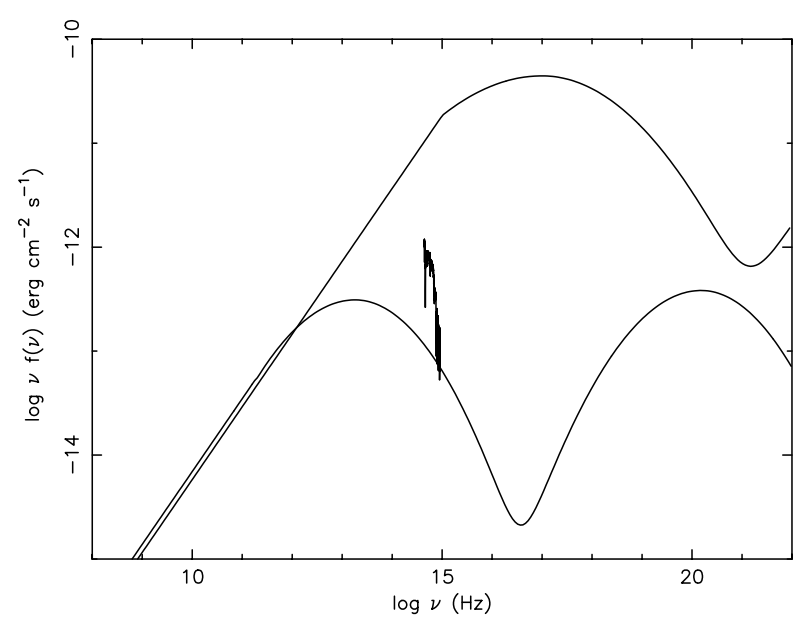

Fig. 6. Typical HBL and LBL spectral energy distributions compared to the SED of a typical giant elliptical galaxy. Note that the emission from the host galaxy is much more easily washed out by an HBL than by a LBL for the same radio flux.

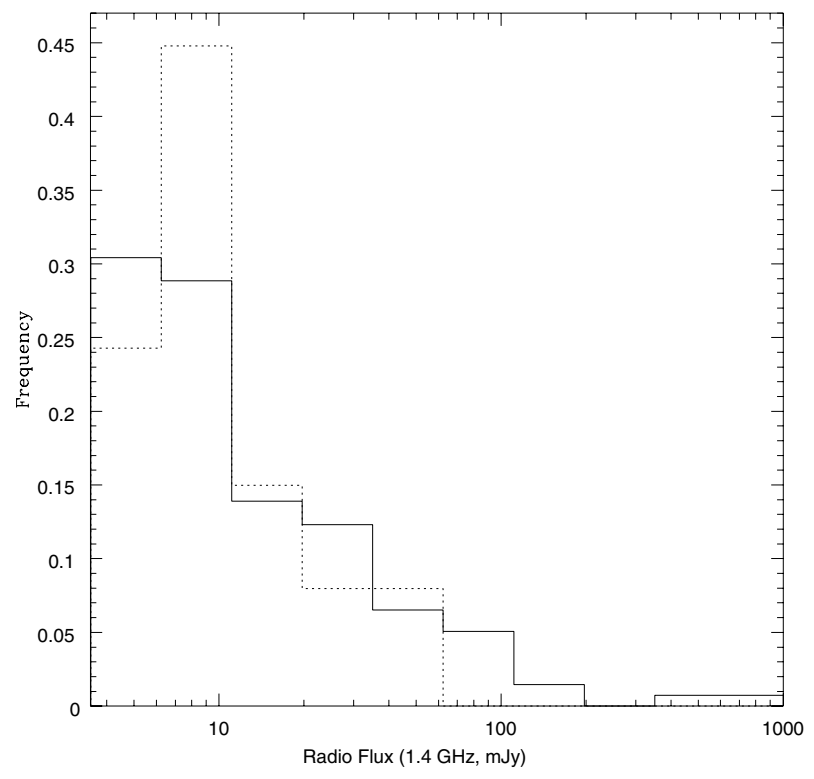

Fig. 7. The sky coverage corrected distributions of $1.4 \mathrm{GHz}$ radio fluxes for BL Lacs with measured redshift (solid line) and for the subsample of objects where the redshift could not be measured due to the featureless nature of the optical spectrum (dotted line).

In principle these objects could also be very bright nearby radio sources; however if that were the case their radio flux distribution would be heavily biased towards high fluxes compared to that of the subsample of objects with a measured redshift. In Fig. 7 the distributions of $1.4 \mathrm{GHz}$ radio fluxes for BL Lacs with measured redshift (solid line) and for the subsample of objects where the redshift could not be measured due to the featureless nature of the optical spectrum (dotted line) are shown. As can be seen, the latter objects strongly peak at low radio flux values indicating that they cannot be nearby very high luminosity objects that out-shine the host galaxy but rather high redshift high luminosity sources.

The only other alternative is that the luminosity of the host galaxies of these objects is extremely low contrary to the findings of Wurtz et al. (1997) and Urry et al. (2000).
We conclude that it is highly probable that the featureless objects are high redshift-high luminosity High Energy Peaked BL Lacs.

\subsection{Rejected broad-lined candidates}

The multi-frequency statistical selection criteria of the Sedentary survey have been estimated to be about $85 \%$ efficient. This paragraph deals with those candidates that although satisfied all the selection conditions of Paper I were at some point excluded from the sample because they were either reported in the literature as known emission line AGN or were not confirmed as BL Lacs by the optical identification process.

Specifically, in 8 cases the candidate HBLs have been found to be known emission line AGN and were rejected in Paper I; other 3 candidates have been identified as emission line AGN by Bauer et al. (2000) and Schwope et al. (2000) after the publication of Paper I, while our optical spectroscopic campaign revealed 8 emission line AGN out of the 58 previously unclassified candidates that were observed.

All together the classification of all candidates in the Sedentary Survey revealed that only 19 out of the original 163 objects in the "HBL zone" showed emission lines that are too strong for a source to be called BL Lacertae object according to the classification method of Marchã et al. (1996). The total level of contamination is therefore about $12 \%$, well within the expected value of $\sim 15 \%$ (see Paper I).

The 19 rejected emission line AGNs are listed in Table 3 where Col. 1 gives the RASS name, Cols. 2-4 the fluxes in the X-ray $(0.1-2.4 \mathrm{keV})$, radio $(20 \mathrm{~cm}$, from the NVSS survey) and the optical apparent $V$ magnitude (from APM and COSMOS, see Paper I) respectively, Col. 5 the redshift, Cols. 6 and 7 give the radio luminosity and the $\alpha_{\mathrm{ox}}$; Col. 8 gives the reference for the optical identification.

These X-ray sources are mostly nearby, low radio luminosity emission line AGN with $\alpha_{\text {ro }}$ close to the selection threshold of 0.2 . The radio emission of these sources is probably of nonnuclear origin and the X-ray emission may be unrelated to it.

From previous surveys we know that FSRQs with low $f_{\mathrm{x}} / f_{\mathrm{r}}$ are much more abundant than BL Lacs of similar $f_{\mathrm{x}} / f_{\mathrm{r}}$ both at high (e.g. in the 1 Jy sample Stickel \& Kühr 1993; Stickel et al. 1991) and lower radio flux (e.g. $50 \mathrm{mJy}$, in the DXRBS survey Padovani et al. 1997; Landt et al. 2001). Until recently X-ray strong (high $f_{\mathrm{x}} / f_{\mathrm{r}}$ ) FSRQ or HFSRQs (High energy peaked FSRQs) were instead thought to be very rare or altogether non existent; however Padovani et al. (2003) discovered that a relative large number of HFSRQs indeed exist, although none with $f_{\mathrm{x}} / f_{\mathrm{r}}$ values as high as those required for inclusion in the sedentary survey.

If the relative abundance of FSRQ and BL Lacs were independent of $f_{\mathrm{x}} / f_{\mathrm{r}}$ (or, equivalently, on the position of the synchrotron peak energy) our survey, that includes 150 BL Lacs, should have detected several hundred extreme HFSRQs. Since we have possibly found only very few cases, all at relatively low redshifts and low radio power, we confirm with high statistical confidence that FSRQs very rarely (if at all) reach $f_{\mathrm{x}} / f_{\mathrm{r}}$ values so high as to satisfy condition 2 ) in our survey definition 
criteria $\left(f_{\mathrm{x}} / f_{\mathrm{r}} \geq 3 \times 10^{-10} \mathrm{erg} \mathrm{cm}^{-2} \mathrm{~s}^{-1} \mathrm{Jy}^{-1}\right)$. In other words the synchrotron component in broad lined Blazars do not reach peak energies as high as those found in HBL BL Lacs.

\section{X-ray spectroscopy with BeppoSAX}

The BeppoSAX X-ray Astronomy Satellite (see Boella et al. 1997 for a full description) was characterized by a very wide bandpass $(0.1 \sim 200 \mathrm{keV})$ and therefore particularly well suited to study the X-ray spectrum of bright extragalactic objects like HBL BL Lacs. BeppoSAX successfully operated for a period of six years, from May 1996 to May 2002, during which it accumulated a large data archive, unique in terms of bandwidth, that can be used to determine the broad band X-ray spectrum of these sources.

A compilation of all the BeppoSAX spectral data for Blazars which were publicly available in March 2002 has been presented in Giommi et al. (2002a). In the following we update this work concentrating on the subset of objects belonging to the Sedentary survey and we complete the dataset by adding all the observations carried out after March 2002.

Twenty five objects of the Sedentary survey have been observed with BeppoSAX as part of different observing programs for a total of 50 pointings. Giommi et al. (2002a) considered several spectral models to fit the data and concluded that for HBL BL Lacs, which very often exhibit continuous convex curvature in the X-ray band, the model that best matches the data is a logarithmic parabola of the type

$F(E)=K\left(E / E_{*}\right)^{-\left(a+b * \log \left(E / E_{*}\right)\right)} \quad\left(\mathrm{ph} / \mathrm{cm}^{2} / \mathrm{s} / \mathrm{keV}\right)$.

This model is particularly appealing since it can describe broad-band spectral curvature with only three free parameters $K, a$ and $b$, just one more than a simple power law; in the following we assume $E_{*}=1 \mathrm{keV}$. Massaro et al. (2004a) and Massaro et al. (2004b) carefully analyzed all the BeppoSAX observations of the bright HBL BL Lacs Mkn 421 and Mkn 501 and showed that the log-parabolic model is a good representation of the spectrum of these sources in all intensity states over the entire BeppoSAX energy bandpass. These authors also showed that the parameters of the logarithmic parabola model may provide information on the particle acceleration mechanism and can be used to easily calculate useful quantities such as an energy dependent photon index $\Gamma(E)$ :

$\Gamma(E)=a+2 b \log \left(E / E_{*}\right)$.

The parameter $a$ is the photon index at the energy $E_{*}$, while $b$ measures the curvature of the parabola. The peak frequency $v_{\mathrm{p}}=E_{\mathrm{p}} / h$, corresponding to the maximum in the $v-v F(v)$ plot, is given by:

$E_{\mathrm{p}}=E_{*} 10^{(2-a) / 2 b}(\mathrm{keV})$

and the maximum value is:

$\nu_{\mathrm{p}} F\left(\nu_{\mathrm{p}}\right)=\left(1.60 \times 10^{-9}\right) K E_{*}^{2} 10^{(2-a)^{2} / 4 b} \mathrm{erg} /\left(\mathrm{cm}^{2} \mathrm{~s}\right)$,

(Massaro et al. 2004a).

The X-ray data analysis was carried out in a uniform way using the XANADU package (XIMAGE,

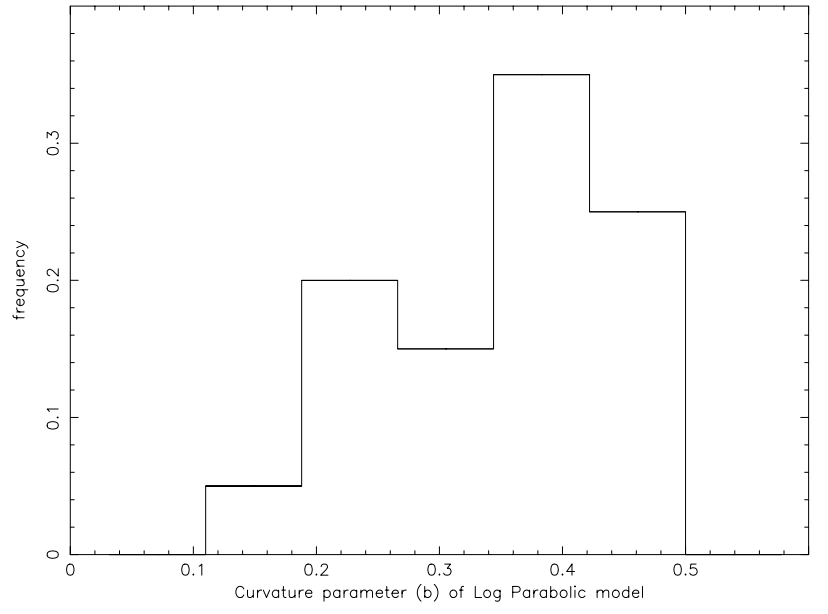

Fig. 8. The distributions of the curvature parameter $(b)$ in the $\log$ parabolic model for the subsample of objects (18) for which the parameter $b$ could be estimated with a statistical error less than 0.25 .

XRONOS, XSPEC) and following the standard procedures described in the BeppoSAX documentation (http://www.asdc.asi.it/bepposax/). Spectral fitting was carried out with XSPEC (v. 11.0) using the calibration files available from the BeppoSAX Science Data Center. We have used data from the LECS and MECS experiments and, whenever the source was bright enough we also included the high energy PDS data. The results are reported in Table 4 where Col. 1 gives the source name, Col. 2 gives the date of the BeppoSAX observation, Col. 3 gives the instruments used for the analysis; Cols. $4-6$ give the best fit parameters ( $a b$ and $K$ ) together with 1 sigma errors, Col. 7 gives the reduced $\chi^{2}$, Col. 8 gives the $2-10 \mathrm{keV}$ flux in units of $10^{-11} \mathrm{erg} \mathrm{cm}^{-2} \mathrm{~s}^{-1}$ and Col. 9 gives the peak energy derived using Eq. (3).

As can be seen, in all but a few cases (see notes below), the wide band X-ray spectra of the objects in the Sedentary Survey observed by BeppoSAX are satisfactorily described by the logparabolic model. The curvature parameter $(b)$ has been found to be positive, that is the spectrum is downward curved, in all sources with the exception of SHBL J020106.6+003401 and SHBLJ 123511.0-140322 (observation of July 1999) where the curvature estimation is very uncertain. The distribution of $b$ is shown in Fig. 8 where it can be seen that the values of the curvature parameter measured with a reasonable accuracy cluster around values of $b \approx 0.4$ with a hint of a secondary peak at $b \approx 0.2$. This second peak, however is not statistically significant and larger samples would be needed to confirm or disprove it.

The energy where the emitted power is maximum $\left(E_{\mathrm{p}}\right)$ can be calculated from $b$ and the measured photon index at $1 \mathrm{keV}$ (a) using Eq. (3). In Fig. 9 the two quantities are plotted against each other. In this plot we have added the best fit values for Mkn 501 from Massaro et al. (2004b) to extend the $E_{\mathrm{p}}$ range to about $100 \mathrm{keV}$. The plotted quantities should be on a straight line if the curvature parameter were the same in all objects. From Fig. 9 we see that most of the sources actually lie along the $b=0.4$ line in most intermediate cases $\left(0.1<E_{\text {peak }}<5 \mathrm{keV}\right)$ but significant deviations are apparent 
Table 4. Results of BeppoSAX spectral fittings.

\begin{tabular}{|c|c|c|c|c|c|c|c|c|}
\hline \multirow{3}{*}{$\begin{array}{l}\text { Source name } \\
\text { SHBL J }\end{array}$} & \multirow{3}{*}{$\begin{array}{l}\text { Observation } \\
\text { date } \\
(2)\end{array}$} & \multirow{3}{*}{$\begin{array}{c}\text { Instrument(s) } \\
\text { used }^{(*)} \\
\text { (3) }\end{array}$} & \multicolumn{3}{|c|}{ Best fit parameters } & \multirow{3}{*}{$\begin{array}{c}\chi_{r}^{2} \text { (d.o.f.) } \\
\text { (7) }\end{array}$} & \multirow{3}{*}{$\begin{array}{l}\text { flux }^{(a)} \\
(8)\end{array}$} & \multirow{3}{*}{$\begin{array}{c}E_{\mathrm{p}} \\
(\mathrm{keV}) \\
(9)\end{array}$} \\
\hline & & & $a$ & $b$ & $K$ & & & \\
\hline & & & (4) & (5) & (6) & & & \\
\hline \multirow[t]{2}{*}{$012308.7+342049$} & $03-01-99$ & $\mathrm{~L} \mathrm{M}$ & $1.9 \pm 0.1$ & $0.2 \pm 0.1$ & $(6.7 \pm 0.6) 10^{-3}$ & $1.1(49)$ & 1.70 & 1.8 \\
\hline & 02-02-99 & L M & $1.8 \pm 0.1$ & $0.4 \pm 0.1$ & $(6.0 \pm 0.7) 10^{-3}$ & $0.89(42)$ & 1.30 & 1.8 \\
\hline $013632.5+390559$ & 09-01-01 & $\mathrm{L} \mathrm{M}$ & $2.0 \pm 0.1$ & $0.5 \pm 0.1$ & $(6.6 \pm 0.4) 10^{-3}$ & $1.11(44)$ & 1.04 & 1.0 \\
\hline $020106.3+003401$ & $16-08-96$ & $\mathrm{~L} \mathrm{M}$ & $2.5 \pm 0.3$ & $-0.2 \pm 0.3$ & $(1.6 \pm 0.7) 10^{-3}$ & $1.25(20)$ & 0.26 & $\ldots$ \\
\hline $031951.9+184534$ & $15-01-97$ & L M & $1.6 \pm 0.3$ & $0.4 \pm 0.2$ & $(2.3 \pm 0.7) 10^{-3}$ & $0.75(40)$ & 0.7 & 3.2 \\
\hline $032613.9+022515$ & $20-01-98$ & $\mathrm{~L} \mathrm{M}$ & $1.5 \pm 0.3$ & $0.7 \pm 0.4$ & $(1.2 \pm 0.6) 10^{-3}$ & $0.95(20)$ & 0.3 & 2.3 \\
\hline $034923.2-115927$ & $10-01-97$ & L M & $1.7 \pm 0.2$ & $0.4 \pm 0.2$ & $(2.4 \pm 0.6) 10^{-3}$ & $0.99(41)$ & 0.6 & 2.4 \\
\hline $041652.4+010524$ & $21-09-96$ & M & $2.3 \pm 0.4$ & $0.3 \pm 0.3$ & $(6.4 \pm 1.8) 10^{-3}$ & $0.76(33)$ & 0.84 & 0.3 \\
\hline $050939.0-040036$ & $11-02-99$ & L M & $1.6 \pm 0.2$ & $0.5 \pm 0.2$ & $(2.0 \pm 0.5) 10^{-3}$ & $0.90(35)$ & 0.55 & 2.5 \\
\hline $074405.6+743358$ & $29-10-96$ & L M & $2.5 \pm 0.9$ & $0.2 \pm 0.7$ & $(1.3 \pm 1.3) 10^{-3}$ & $1.50(17)$ & 0.14 & 0.1 \\
\hline $093037.5+495025$ & $25-11-98$ & $\mathrm{~L} \mathrm{M}$ & $1.7 \pm 0.1$ & $0.3 \pm 0.1$ & $(2.3 \pm 0.4) 10^{-3}$ & $1.04(42)$ & 0.66 & 3.2 \\
\hline $103118.6+505335$ & $01-05-97$ & $\mathrm{~L} M$ & $2.1 \pm 0.1$ & $0.3 \pm 0.1$ & $(6.2 \pm 0.3) 10^{-3}$ & $0.75(42)$ & 1.02 & 0.7 \\
\hline \multirow[t]{2}{*}{ 110337.6-232931 } & 04-01-97 & L M P & $1.6 \pm 0.1$ & $0.3 \pm 0.1$ & $(11.7 \pm 0.8) 10^{-3}$ & $1.22(82)$ & 3.72 & 4.6 \\
\hline & 19-06-98 & L M & $1.9 \pm 0.1$ & $0.3 \pm 0.1$ & $(11.7 \pm 0.6) 10^{-3}$ & $1.20(89)$ & 2.51 & 1.5 \\
\hline \multirow[t]{13}{*}{$110427.3+381231^{(b)}$} & $29-04-97$ & $\mathrm{~L} \mathrm{M}$ & $2.25 \pm 0.01$ & $0.45 \pm 0.01$ & $(7.2 \pm 0.1) 10^{-2}$ & $1.31(132)$ & 8.5 & 0.5 \\
\hline & $30-04-97$ & L M & $2.26 \pm 0.01$ & $0.47 \pm 0.01$ & $(7.3 \pm 0.1) 10^{-2}$ & $0.75(136)$ & 8.3 & 0.5 \\
\hline & $01-05-97$ & L M & $2.23 \pm 0.01$ & $0.43 \pm 0.01$ & $(8.2 \pm 0.1) 10^{-2}$ & $1.06(132)$ & 10.0 & 0.5 \\
\hline & $02-05-97$ & L M & $2.25 \pm 0.01$ & $0.43 \pm 0.02$ & $(10.2 \pm 0.2) 10^{-2}$ & $0.86(127)$ & 12.3 & 0.5 \\
\hline & 03-05-97 & L M & $2.32 \pm 0.02$ & $0.44 \pm 0.02$ & $(6.6 \pm 0.1) 10^{-2}$ & $0.97(100)$ & 7.1 & 0.4 \\
\hline & $04-05-97$ & L M & $2.50 \pm 0.02$ & $0.48 \pm 0.02$ & $(5.0 \pm 0.1) 10^{-2}$ & $1.16(100)$ & 4.1 & 0.3 \\
\hline & $05-05-97$ & L M & $2.40 \pm 0.01$ & $0.45 \pm 0.02$ & $(6.5 \pm 0.1) 10^{-2}$ & $1.11(100)$ & 6.3 & 0.4 \\
\hline & 21-04-98 & L M P & $2.07 \pm 0.004$ & $0.34 \pm 0.006$ & $(18.8 \pm 0.1) 10^{-2}$ & $1.19(171)$ & 31.0 & 0.8 \\
\hline & $23-04-98$ & L M P & $2.22 \pm 0.004$ & $0.37 \pm 0.007$ & $(13.5 \pm 0.1) 10^{-2}$ & $1.02(169)$ & 17.8 & 0.5 \\
\hline & $22-06-98$ & L M P & $2.07 \pm 0.007$ & $0.34 \pm 0.008$ & $(14.4 \pm 0.1) 10^{-2}$ & $1.26(150)$ & 23.9 & 0.8 \\
\hline & 04-05-99 & L M P & $2.42 \pm 0.004$ & $0.42 \pm 0.005$ & $(11.4 \pm 0.1) 10^{-2}$ & $1.11(141)$ & 11.0 & 0.3 \\
\hline & $26-04-00$ & L M P & $1.81 \pm 0.002$ & $0.21 \pm 0.002$ & $(22.7 \pm 0.1) 10^{-2}$ & $1.06(152)$ & 62.5 & 2.8 \\
\hline & $09-05-00$ & L M P & $1.88 \pm 0.003$ & $0.18 \pm 0.003$ & $(19.4 \pm 0.1) 10^{-2}$ & $1.34(149)$ & 49.5 & 2.2 \\
\hline $111706.3+201407$ & $13-12-99$ & $\mathrm{~L} \mathrm{M}$ & $2.44 \pm 0.04$ & $0.48 \pm 0.05$ & $(6.7 \pm 0.3) 10^{-3}$ & $1.43(54)$ & 0.61 & 0.3 \\
\hline $112048.0+421212$ & $01-05-97$ & $\mathrm{~L} \mathrm{M}$ & $2.22 \pm 0.07$ & $0.4 \pm 0.1$ & $(2.3 \pm 0.2) 10^{-3}$ & $0.40(25)$ & 0.28 & 0.5 \\
\hline \multirow[t]{3}{*}{$121158.6+224232$} & $27-12-99$ & $\mathrm{~L} M$ & $1.8 \pm 0.1$ & $0.3 \pm 0.1$ & $(0.9 \pm 0.2) 10^{-3}$ & $1.10(43)$ & 0.24 & 2.2 \\
\hline & $28-12-01$ & L M & $1.62 \pm 0.08$ & $0.24 \pm 0.07$ & $(2.1 \pm 0.2) 10^{-3}$ & $1.03(54)$ & 0.75 & 6.2 \\
\hline & $11-01-02$ & $\mathrm{~L} \mathrm{M}$ & $1.4 \pm 0.2$ & $0.7 \pm 0.2$ & $(1.7 \pm 0.3) 10^{-3}$ & $1.45(41)$ & 0.52 & 2.7 \\
\hline $122121.9+301037$ & $12-07-99$ & L M & $2.10 \pm 0.03$ & $0.37 \pm 0.03$ & $(9.5 \pm 0.3) 10^{-3}$ & $0.78(88)$ & 1.48 & 0.7 \\
\hline \multirow[t]{2}{*}{$123511.0-140322$} & $27-06-99$ & $\mathrm{~L} M$ & $1.4 \pm 0.3$ & $0.9 \pm 0.4$ & $(0.8 \pm 0.2) 10^{-3}$ & $0.56(19)$ & 0.19 & 2.2 \\
\hline & $16-07-99$ & $\mathrm{~L} \mathrm{M}$ & $2.6 \pm 0.2$ & $-0.4 \pm 0.3$ & $(1.0 \pm 0.2) 10^{-3}$ & $0.46(19)$ & 0.15 & $\ldots$ \\
\hline $125731.9+241240$ & $20-06-98$ & $\mathrm{~L} M$ & $1.6 \pm 0.3$ & $0.5 \pm 0.3$ & $(3.8 \pm 1.2) 10^{-3}$ & $0.84(38)$ & 1.11 & 2.5 \\
\hline \multirow[t]{3}{*}{$141756.1+254356$} & $13-07-00$ & $\mathrm{~L} M$ & $1.71 \pm 0.08$ & $0.47 \pm 0.07$ & $(5.2 \pm 0.4) 10^{-3}$ & $1.15(49)$ & 1.26 & 2.0 \\
\hline & $23-07-00$ & $\mathrm{~L} \mathrm{M}$ & $1.5 \pm 0.2$ & $0.7 \pm 0.2$ & $(3.4 \pm 0.9) 10^{-3}$ & $0.84(36)$ & 0.88 & 2.3 \\
\hline & $27-07-00$ & L M & $1.85 \pm 0.08$ & $0.30 \pm 0.07$ & $(4.0 \pm 0.4) 10^{-3}$ & $1.25(49)$ & 0.93 & 1.8 \\
\hline $142832.6+424024$ & 08-02-99 & L M & $1.81 \pm 0.03$ & $0.11 \pm 0.03$ & $(6.6 \pm 0.3) 10^{-3}$ & $0.98(87)$ & 2.03 & 7.3 \\
\hline $151747.4+652523$ & 05-03-97 & $\mathrm{L} \mathrm{M}$ & $2.0 \pm 0.2$ & $0.4 \pm 0.2$ & $(5.5 \pm 0.8) 10^{-3}$ & $1.30(39)$ & 0.98 & 1.0 \\
\hline $153500.9+532037$ & $13-02-99$ & L M & $2.0 \pm 0.3$ & $0.4 \pm 0.3$ & $(1.4 \pm 0.5) 10^{-3}$ & $0.89(38)$ & 0.25 & 1.0 \\
\hline \multirow[t]{3}{*}{$215852.0-301331$} & $20-11-96$ & L M P & $2.42 \pm 0.006$ & $0.23 \pm 0.01$ & $(49.3 \pm 0.4) 10^{-3}$ & $1.22(130)$ & 5.59 & 0.1 \\
\hline & $22-11-97$ & L M P & $2.38 \pm 0.008$ & $0.36 \pm 0.01$ & $(77.6 \pm 0.8) 10^{-3}$ & $1.28(108)$ & 8.28 & 0.3 \\
\hline & 04-11-99 & $\mathrm{L} \mathrm{M}$ & $2.65 \pm 0.009$ & $0.20 \pm 0.01$ & $(28.9 \pm 0.4) 10^{-3}$ & $1.16(103)$ & 2.47 & 0.02 \\
\hline 235907.9-303739 & $21-06-98$ & L M P & $1.78 \pm 0.03$ & $0.25 \pm 0.03$ & $(8.8 \pm 0.3) 10^{-3}$ & $1.35(91)$ & 2.43 & 2.8 \\
\hline
\end{tabular}

(a) X-ray flux in the 2-10 keV band in units of $10^{-11} \mathrm{erg} \mathrm{cm}^{-2} \mathrm{~s}^{-1}$.

(b) Spectral parameters taken from Massaro et al. (2004a).

${ }^{(*)} \mathrm{L}=$ LECS; $\mathrm{M}=$ MECS; P = PDS. 


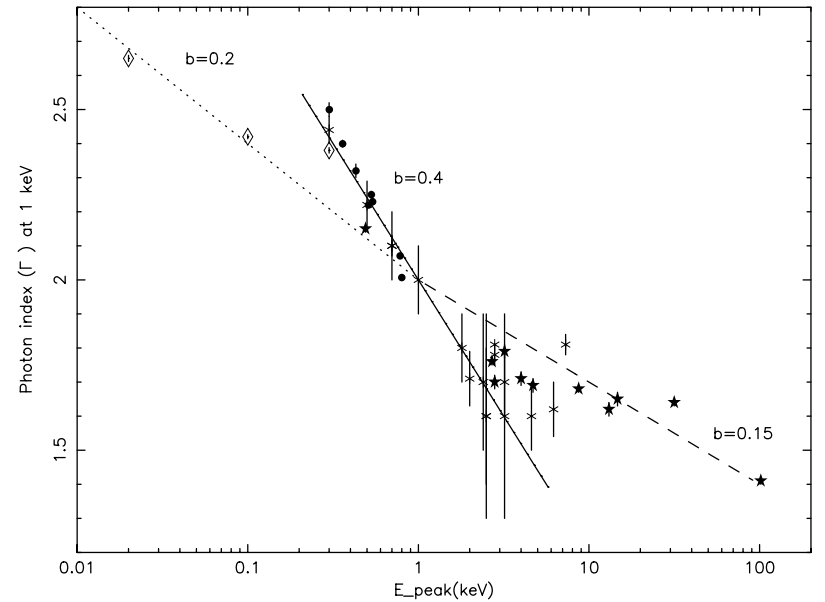

Fig. 9. The power law spectral index $(\Gamma)$ at $1 \mathrm{keV}(a)$ plotted against the peak energy. A strong correlation is present for $E_{\mathrm{p}}$ (as predicted by Eq. (3) if $b$ is constant) values less than about $5 \mathrm{keV}$, above which $\Gamma$ remains approximately constant. Crosses represent most of the objects listed in Table 3, diamonds are for PKS 2155-304, filled circles for Mkn 421 and filled stars for Mkn 501, which is not included in the sedentary survey because at the time of the RASS survey was in a very low state, but was added to this plot to extend the $E_{\mathrm{p}}$ dynamical range to about $100 \mathrm{keV}$. The solid, dotted and dashed lines represent the expected correlations for $b=0.4, b=0.2$ and $b=0.15$, respectively.

both at very low (PKS 2155-304) and at very high (Mkn 421 and Mkn 501 during flares) peak energies.

\subsection{Notes on individual objects}

\subsubsection{SHBL J020106.3+003401}

This is the only object in the sample which shows concave (upward instead of downward) curvature in the BeppoSAX $\mathrm{X}$-ray spectrum. Most of this curvature is due to a feature above $6 \mathrm{keV}$ where the statistics are not good enough to allow us to perform a detailed analysis. Moreover this source is within 3 arcmin from the QSO SDSS J020115.53+003135.1 which is clearly detected both in the BeppoSAX and in a Rosat PSPC image (although at a flux level lower than that of SHBL J020106.3+003401) that could contaminate the BeppoSAX data.

\subsubsection{SHBL J074405.6+743358}

The logarithmic parabola fit to the X-ray spectrum of this source is not a good representation of the data.The reduced $\chi^{2}$ is 1.5 ( 17 d.o.f.) which is possibly due to the poor quality of the data and to a some flux excess above $4-5 \mathrm{keV}$.

\section{Broad-band spectral energy distributions and synchrotron peaks}

One of the main motivations of the Sedentary Survey was the selection of a sizable radio flux limited sample of HBL BL Lacs, a type of sources that in the past have been discovered almost exclusively in X-ray surveys.
The very high $\mathrm{X}$-ray to radio flux ratio that characterizes these objects is thought to be the result of synchrotron radiation extending to X-ray or even higher energies. To verify that this is indeed the case we have constructed the broadband SED (in the usual $\log (v F(v))$ vs. $\log (v)$ space) combining the BeppoSAX X-ray data (de-reddened using the cross sections of Morrison \& McCammon (1983) setting the amount of absorbing material $\left(N_{\mathrm{H}}\right)$ equal to the Galactic value along the line of sight), with the (non-simultaneous) optical spectroscopy data from the sedentary identification campaign when available, and with non-simultaneous multi-frequency literature data taken from NED, from the NVSS $20 \mathrm{~cm}$ survey (Condon et al. 1998), the Two Micron All Sky Survey (2MASS, Skrutskie et al. 1995), the Sloan Digital Sky Survey (SDSS, Sloan Digital Sky Survey 2001), the GSC2 catalog (Lasker 1995; B.J. et al. 2000) and the Rosat All Sky Survey (Voges et al. 1999).

\subsection{MASS and GSC2 fluxes}

The Two Micron All Sky Survey (2MASS) covers the full sky at near infra-red frequencies and allows us to add three flux measurements to our SEDs, at least for the brightest sources in our Survey. Indeed for all objects brighter that $V \sim 17.5 \mathrm{a}$ counterpart in the 2MASS Point Source Catalog (Cutri et al. 2003) has been found.

We have converted $J, H$ and $K$ s magnitudes from the 2MASS survey into monochromatic fluxes at 1.24, 1.66 and 2.16 microns assuming the following calibration (e.g. Cohen et al. 2003)

$$
\begin{aligned}
& f(1.24 \mu)=10^{-0.4\left(m_{J}-15.51\right)} \mathrm{mJy} \\
& f(1.66 \mu)=10^{-0.4\left(m_{H}-15.03\right)} \mathrm{mJy} \\
& f(2.16 \mu)=10^{-0.4\left(m_{K \mathrm{~s}}-14.56\right)} \mathrm{mJy} .
\end{aligned}
$$

GSC2 $F$ and $J$ magnitudes have been converted as follows:

$$
\begin{aligned}
& f\left(4.5510^{14} \mathrm{~Hz}\right)=10^{-0.4\left(m_{F}-16.16\right)} \mathrm{mJy} \\
& f\left(6.0010^{14} \mathrm{~Hz}\right)=10^{-0.4\left(m_{J}-16.43\right)} \mathrm{mJy},
\end{aligned}
$$

all magnitudes have been de-reddened according to the prescriptions of Cardelli et al. (1989).

\subsection{SED shapes}

The SEDs of all objects for which BeppoSAX data are available are plotted in Figs. 10 through 34; for reasons of brevity the SED for the remaining objects, which include only a few points, are not plotted here but are available on-line at the following web site http://www .asdc.asi.it/sedentary/ and at http://www. edpsciences.org/. Although the data are not simultaneous, the $v f(v)$ dynamical range $(\approx$ a factor of 1000) is much larger than the expected variability and allows us to characterize the broad band spectrum of HBL BL Lacs comparing the observed energy distribution to the expectations of a homogeneous Synchrotron Self-Compton (SSC) 
model adapted from Tavecchio et al. (1998). This model assumes that radiation is produced by a population of relativistic electrons emitting synchrotron radiation in a single zone of a jet that is moving at relativistic speed and at a small angle with respect to the line of sight. These photons are subsequently scattered by the same electrons to higher energies via the inverse Compton process (ignoring the Comptonization of external photons, which only affects the SED at $\gamma$-ray energies; e.g., Ghisellini et al. 1998). The physical parameters that define the model are the jet radius, the Doppler factor, the magnetic field B, and four spectral parameters of the electron population, assumed to follow a power-law distribution which turns into a log parabolic distribution above a given energy: the normalization, the two spectral slopes, and the break energy. The Klein-Nishina cross section is used in the computation of the Compton scattering.

In a number of cases (e.g. SHBLJ012308.7+342049, SHBLJ041652.4+010524 SHBLJ103118.6+505335 etc.) the $\mathrm{X}$-ray spectral data is consistent with being the smooth extrapolation of the same synchrotron emission observed at radio and optical frequencies, as predicted by a homogeneous single zone SSC model. In other cases (e.g. SHBLJ0301951.9+184534, SHBLJ032613.9+022515, SHBLJ142832.6+424024 etc.), although the X-ray intensity is approximately located on the prediction of homogeneous SSC models, the low energy backextrapolation of the BeppoSAX data clearly falls below the observed optical emission. This has been been also noted in the detailed analysis of several BeppoSAX observations of Mkn 421 and Mkn 501 (Massaro et al. 2004a,b) who interpreted this as due to the superposition of different emission components, the most energetic one possibly due to highly energetic electrons located in a very compact region inside the jet. Because of its small size the power output of this high energy component is only a fraction of the overall emission at optical frequencies but it becomes the dominant emission at very high energies where all other components have dropped well below their peak emission.

\section{Discussion}

We have presented the final, cleaned sample of the "Sedentary Survey of extreme HBL BL Lacs" which comprises 150 sources and is currently the deepest, largest, statistically complete and $100 \%$ identified flux-limited sample of BL Lacertae objects.

By means of multi-frequency literature data, our own optical spectroscopic observations and of wide band X-ray data from the BeppoSAX public archive we have investigated the local and the broad-band spectral properties of the sample.

We have found that:

1. The completion of the optical spectroscopy campaign, which led to the identification of all the objects in the survey (Paper III), fully confirmed the original assumption that a very high percentage of the sources in the initial sample were indeed BL Lac objects. In fact, 50 out of 58 previously unidentified candidates observed by us, or $86 \%$, turned out to be BL Lacs, a similar percentage was found in the sources identified as part of different programs. This result validates the correctness of the selection method and the robustness of the preliminary results reported in Giommi et al. (1999) and Perri et al. (2002). The revised results on the statistical properties of the sample, including $\log N-\log S$, luminosity function and cosmological evolution, will be presented in a separate paper (Paper IV).

2. A significant fraction of the sources $(\sim 25 \%)$ displays a smooth non-thermal optical continuum without features due to either narrow emission lines or to the host galaxy. No redshift or luminosity can therefore be derived for these objects. However, since this condition only occurs when the nuclear non-thermal emission is much brighter than that of the host galaxy, a lower limit to the luminosity can be obtained setting the luminosity of the BL Lac equal to that of the host galaxy which is typically a giant elliptical with approximately constant absolute magnitude (e.g. Wurtz et al. 1997; Urry et al. 2000). Unless these sources are all associated with (never observed) very low luminosity hosts they must be the most luminous objects in the survey. An independent indication that these sources are likely to be high redshift objects is provided by the progression of the dominance of the non-thermal over the galaxian component with redshift shown in Fig. 3 and by the distribution of radio fluxes shown in Fig. 7.

We conclude that the $25 \%$ of featureless sources in the sample are very likely intrinsically bright and therefore probably represent plentiful examples of the yet unreported high radio luminosity-high energy peaked BL Lacs. However, how luminous it remains to be determined. The existence of these sources would be at variance with the claimed inverse proportionality between radio power and synchrotron peak energy known as the "Blazar sequence".

This luminosity sequence (Fossati et al. 1998), which was interpreted as the direct consequence of differential cooling efficiencies in low and high radio power objects (Ghisellini et al. 1998), was based on the absence of low radio powerlow $v_{\text {peak }}$ and of high radio power-high $v_{\text {peak }}$ objects in a composite sample of BL Lacs and FSRQs detected in X-ray and radio flux limited surveys which, although each complete above its flux limit, probed widely different radio luminosity regimes.

The "Blazar sequence" has been recently tested using new deeper, larger and more homogeneous (i.e. with a single flux limit) samples of Blazars. The existence of low-radio luminosity-low $v_{\text {peak }}$ objects has been demonstrated by Padovani et al. (2003) and Caccianiga \& Marchã (2004) who also showed that these are core-dominated radio sources just like the other BL Lacs and that their low radio power cannot be explained as the consequence of large orientation angles. In addition, the existence of high radio luminosity-high $v_{\text {peak }}$ sources has been discussed by Giommi et al. (2002b) on the basis of preliminary results of the Sedentary Survey.

3. The relative abundance of FSRQ and BL Lacs of any $f_{\mathrm{x}} / f_{\mathrm{r}}$ flux ratio is approximately of three-four FSRQ for each BL Lac (e.g. Stickel et al. 1991; Landt et al. 2001). Although the selection criteria of the Sedentary Survey 
do not preclude the detection of FSRQs, we have found 150 extreme HBL BL Lacs and almost no FSRQs, instead of several hundred if the relative abundance of strongly lined and line-less Blazars were independent of $f_{\mathrm{x}} / f_{\mathrm{r}}$. Although a number of intermediate FSRQs have recently been found by Padovani et al. $(2002,2003)$ it is clear that broad lined Blazars do not reach the very high synchrotron peak energies of HBL BL Lacs.

4. A few bright nearby elliptical galaxies have been found below the radio loud-radio quiet border $\left(\alpha_{\mathrm{ox}}=0.2\right.$, see Table 2). These objects would normally be labelled "radio galaxies" or "normal ellipticals" and therefore removed from BL Lacs samples. However, these objects, which generally appear as point-like sources in the NVSS, may also be faint HBL BL Lacs whose non-thermal optical component is simply below the emission from the host galaxy. This situation must be expected towards the low end of the radio luminosity function.

In addition, strong flux variability, one of the defining properties of BL Lacs, and the position of the synchrotron peak (see Fig. 6), both have a strong influence on the ratio between the galactic and non-thermal optical flux, blurring the border between BL Lacs and radio galaxies. For example a borderline source that varied its non-thermal flux by a significant amount would be classified as a BL Lac when in a high state and as a radio galaxy when in the low state. Also, as shown in Fig. 6 two BL Lacs with identical radio flux but with synchrotron peaks at low and high energy (e.g. LBL and HBL) would also be classified differently depending on the position of the synchrotron peak.

5. Massaro et al. (2004a) and Massaro et al. (2004b) have shown that the wide band X-ray spectrum of the bright HBL BL Lacs like Mkn 421 and Mkn 501 can be satisfactorily described by a log-parabolic model in all intensity states (see Eq. (1)). We have then fit this model to the X-ray spectrum of all the objects in the survey that have been observed by BeppoSAX obtaining statistically acceptable fits in all but a few cases (see Table 4 ). The curvature parameter $(b)$ has been found to be positive (i.e. the spectrum is downward curved) in almost all sources.

6. The broad band SEDs confirm that these objects are HBLs, that is their synchrotron emission extends to very high energies, sometimes well into the X-ray band. The X-ray data are generally located on the extrapolation for the radio and optical spectral distribution as predicted by homogeneous SSC models with the synchrotron power peaking at X-ray frequencies. However, the local strong X-ray curvature in a number of objects is not consistent with a simple backextrapolation to optical frequencies suggesting that there may be more than one emission component.

7. The particularly energetic physical conditions that are necessary to produce the very high energy synchrotron photons observed imply that the corresponding Inverse Compton radiation must reach energies close to or within the $\mathrm{TeV}$ band. Indeed, even the source with the lowest synchrotron peak energy reported in Table 3 (i.e. PKS 2155-304, $v_{\text {peak }}=0.02-0.3 \mathrm{keV}$ ) has been detected at $\mathrm{TeV}$ frequencies (Chadwick et al. 1999; Hinton 2003). It is therefore natural to expect that many of the sources in the Sedentary survey are $\mathrm{TeV}$ emitters and that the brightest and closest ones may be detectable by the present generation of Cherenkov telescopes, especially during flares. However, despite this obvious prediction only 3 (Mkn $421=$ SHBL J110427.3+381231, PKS 2155-304 = SHBL J215852.0301331 and $\mathrm{H} 1426+428=$ SHBL J142832.6+424024) of the 6 presently established TeV BL Lacs are actually included in our survey. Mkn 501, 1ES2344+514 and 1ES1959+650, the remaining TeV Blazars, all displayed synchrotron peak energies well into the hard X-ray band (Massaro et al. 2004b; Giommi et al. 2000; Krawczynski et al. 2004) during strong outbursts but never reached (not even during the strongest flare) a soft X-ray flux high enough to meet the $f_{\mathrm{x}} / f_{\mathrm{r}}$ condition necessary to be part of the Sedentary survey. Most of the X-ray variations in these sources were in fact confined to the hard X-ray band, close to the maximum of their synchrotron power and above the Rosat bandpass. This implies that synchrotron peak energies in the hard X-ray band are not necessarily located on the smooth extrapolation of the lower energies spectrum, but may be due to additional, very energetic emission components that only emerge above the Rosat X-ray band. The existence of such sub-components is consistent with the observed difference between the shape of the overall SED and the local X-ray spectral curvature in many objects (see Figs. 13, 14, 17, 22, 28, 30 and 31).

It is likely that $\mathrm{TeV}$ emission is associated with these components, some of which may not always be detectable at soft X-ray frequencies because they could be outshone by the main or by some other less energetic synchrotron component. This would be consistent with the existence of the recently reported "X-ray orphan" TeV flares (Krawczynski et al. 2004), that is $\mathrm{TeV}$ flare emission not correlated to soft $\mathrm{X}$-ray flares and that this lack of correlation should be expected in other objects. Simultaneous sensitive hard-X-ray observations are obviously desirable and could be achieved in the short term by organizing $\mathrm{TeV}$ observations with the Swift spacecraft and on the medium term with the next generation of hard X-ray imaging telescopes.

Acknowledgements. This work is partly based on BeppoSAX X-ray data taken from the BeppoSAX public archive hosted at the ASI Science Data Center (ASDC), Frascati, Italy, and on Optical spectroscopy observations performed at the European Southern Observatory, La Silla, Chile, (Proposals ESO Nos. 67.B-0222(A), 71.B-0582(A) and 71.B-0582(B)), Telescopio Nazionale Galileo, La Palma, Canarian Islands (proposals AOT5/02A, AOT6/02B, AOT7/03A) and Kitt Peak National Observatory. This research has also made use of data taken from the following on-line services, the NASA/IPAC Extragalactic Database (NED), the ESO on-line Digitized Sky Survey, the Two Micron All Sky Survey, a joint project of the University of Massachusetts and IPAC, funded by NASA and NSF, the Sloan Digital Sky Survey archive, which is funded by the Alfred P. Sloan Foundation, the Guide Star Catalog-II, which is a joint project of the Space Telescope Science Institute and the Osservatorio Astronomico di Torino.

The authors are grateful to E. Massaro for useful suggestions. 


\section{References}

Anderson, S. F., Voges, W., Margon, B., et al. 2003, AJ, 126, 2209

Bade, N., Beckmann, V., Douglas, N. G., et al. 1998a, A\&A, 334, 459

Bade, N., Engels, D., Voges, W., et al. 1998b, A\&AS, 127, 145

Bade, N., Fink, N., \& Engel, D. 1994, A\&A, 286, 381

Bauer, F., Condon, J., Thuan, T., \& Broderick, J. 2000, ApJS, 129, 547

Beckmann, V. 2000, Ph.D. Thesis, Hamburg University, http://www. sub.uni-hamburg.de/disse/330/vbdiss.html

Beckmann, V., Bade, N., \& Wucknitz, O. 1999, A\&A, 352, 395

Beckmann, V., Engels, D., Bade, N., \& Wucknitz, O. 2003, A\&A, 401, 927

Boella, G., Chiappetti, L., Conti, G., et al. 1997, A\&AS, 122, 327

Bohringer, H., Voges, W., Huchra, J., et al. 2000, ApJS, 129, 435

Buchalter, A., Helfand, D., Becker, R., \& White, R. 1998, ApJ, 494, 503

Caccianiga, A., \& Marchã, M. J. 2004, MNRAS, 348, 937

Cao, L., Wey, J.-Y., \& Hu, J.-Y. 1999, A\&AS, 135, 243

Cardelli, J. A., Clayton, G. C., \& Mathis, J. S. 1989, ApJ, 345, 245

Chadwick, P., Lyons, K., McComb, T., et al. 1999, ApJ, 513, 161

Cohen, M., Wheaton, W. A., \& Megeath, S. T. 2003, AJ, 126, 1080

Condon, J. J., Cotton, W. D., Greisen, E. W., et al. 1998, AJ, 115, 1693

Cutri, R., Skrutskie, M., van Dyk, S., et al. 2003, Univ. of Massachussetts and IPAC on-line catalog.

De Vaucouleurs, G., De Vaucouleurs, A., Corwin, H. G., Jr., et al. 1991, Third Reference Catalogue Of Bright Galaxies, Version 3.9

Falomo, R., Pesce, J., \& Treves, A. 1993, ApJ, 411, 63

Fisher, J., Hasinger, G., Schwope, A., et al. 1998, Astron. Nachr., 319, 347

Fossati, G., Maraschi, L., Celotti, A., Comastri, A., \& Ghisellini, G. 1998, MNRAS, 299, 433

Ghisellini, G., Celotti, A., Fossati, G., Maraschi, L., \& Comastri, A. 1998, MNRAS, 301, 451

Giommi, P., Menna, M. T., \& Padovani, P. 1999, MNRAS, 310, 465

Giommi, P., Padovani, P., \& Perlman, E. 2000, MNRAS, 317, 743

Giommi, P., Capalbi, M., Fiocchi, M., et al. 2002a, in Blazars Astrophysics with BeppoSAX and Other Observatories, ed. P. Giommi, E. Massaro, \& G. Palumbo, 63

Giommi, P., Padovani, P., Perri, M., Landt, H., \& Perlman, E. 2002b, in Blazars Astrophysics with BeppoSAX and Other Observatories, ed. P. Giommi, E. Massaro, \& G. Palumbo, 133

Giommi, P., Perri, M., Piranomonte, S., \& Padovani, P. 2002c, in Blazars Astrophysics with BeppoSAX and Other Observatories, ed. P. Giommi, E. Massaro, \& G. Palumbo, 123

Giommi, P., Piranomonte, S., Perri, M., \& Padovani, P. 2005, in preparation

Hewett, P., Foltz, C., \& Chaffee, F. 1995, AJ, 109, 1498

Hinton, J. 2003, for the H.E.S.S. Collaboration, 2nd VERITAS Symp., Chicago

Irwin, M., Maddox, S., \& McMahon, R. G. 1994, Spectrum, 2, 14

Krawczynski, H., Hughes, B., Horan, D., et al. 2004, ApJ, 601, 151

Landt, H., Padovani, P., \& Giommi, P. 2002, MNRAS, 336, 945

Landt, H., Padovani, P., Perlman, E. S., et al. 2001, MNRAS, 323, 757
Lasker, B. M., McLean B. J., Jenkner, H., Lattanzi, M. G., Spagna, A. 1995, in ESA SP-379, Proc. Symp. Future Possibilities for Astrometry in Space, ed. M. A. C. Perryman \& F. van Leeuwen, 137

Marchã, M. J. M., Browne, I. W. A., Impey, C. D., \& Smith, P. S. 1996, MNRAS, 281, 425

Massaro, E., Perri, M., Giommi, P., \& Nesci, R. 2004a, A\&A, 413, 489

Massaro, E., Perri, M., Giommi, P., Nesci, R., \& Verrecchia, F. 2004b, A\&A, 422, 103

McLean, B. J., Greene, G. R., Lattanzi, M. G., \& Pirenne, B. 2000, in ASP Conf. Ser., ADASS IX, ed. N. Manset, C. Veiller \& D. Crabtree, 216, 145

Morrison, R., \& McCammon, D. 1983, ApJ, 270, 119

NED Redshift, T. 1992, The NED Team Redshift obtained from literature by the NED TEAM prior to November 1992

Padovani, P., Costamante, L., Ghisellini, G., Giommi, P., \& Perlman, E. 2002, ApJ, 581, 895

Padovani, P., \& Giommi, P. 1995, MNRAS, 277, 1477

Padovani, P., Giommi, P., \& Fiore, F. 1997, MmSAI, 68, 147

Padovani, P., Perlman, E., Landt, H., Giommi, P., \& Perri, M. 2003, ApJ, 588, 128

Perri, M., Giommi, P., Piranomonte, S., \& Padovani, P. 2002, in Blazars Astrophysics with BeppoSAX and Other Observatories, ed. P. Giommi, E. Massaro, \& G. Palumbo, 119

Piranomonte, S., Perri, M., Giommi, P., Padovani, P., \& Landt, H. 2004, A\&A, submitted

Polomsky, E., Vennes, S., Thorstensen, J., Mathioudakis, M., \& Falco, E. 1997, ApJ, 486, 179

Puchnarewicz, E., Mason, K., Cordova, F., et al. 1992, MNRAS, 256, 589

Rector, T. A., Stocke, J. T., Perlman, E. S., Morris, S. L., \& Gioia, I. M. 2000, AJ, 120, 1626

Reimers, D., Koehler, T., \& Wisotzki, L. 1996, A\&AS, 115, 235

Schachter, J., Stocke, J., Perlman, E., et al. 1993, ApJ, 412, 541

Schneider, D. P., Schmidt, M., \& Gunn, J. 1994, AJ, 107, 1245

Schwope, A., Hasinger, G., Lehmann, I., et al. 2000, Astron. Nachr., 321,1

Skrutskie, M. F., Beichman, C., Capps, R., et al. 1995, BAAS, 27, 1392

Sloan Digital Sky Survey, T. 2001, Early release as obtained in June 5, 2001, Sloan Digital Sky Survey

Stickel, M., Fried, J. W., Kühr, H., Padovani, P., \& Urry, C. M. 1991, ApJ, 374, 431

Stickel, M., \& Kühr, H. 1993, A\&AS, 101, 521

Urry, C. M., \& Padovani, P. 1995, PASP, 107, 803

Urry, C. M., Scarpa, R., O'Dowd, M., et al. 2000, ApJ, 532, 816

Voges, W., Aschenbach, B., Boller, T., et al. 1999, A\&A, 349, 389

White, R. L., Becker, R. H., Gregg, M., et al. 2000, ApJS, 126, 133

Wurtz, R., Stocke, J. T., Ellingson, E., \& Yee, H. K. C. 1997, ApJ, 480, 547

Yentis, D., Cruddace, R., \& Gursky, H. 1992, in Digitised Optical Sky Surveys, ed. H. MacGillivray \& E. Thomson (Dordrecht: Kluwer Academic Publishers), 67 
P. Giommi et al.: The sedentary survey of extreme high energy peaked BL Lacs. II., Online Material p 1

\section{Online Material}


P. Giommi et al.: The sedentary survey of extreme high energy peaked BL Lacs. II., Online Material p 2

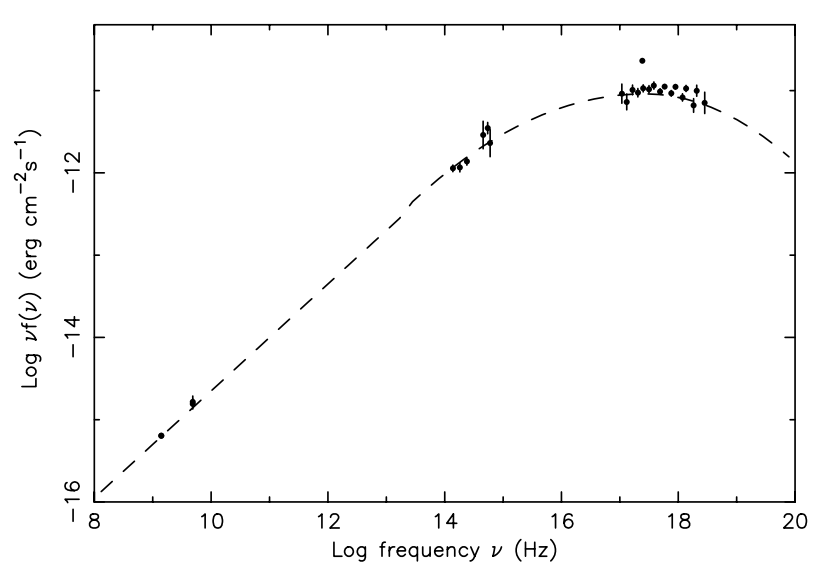

Fig. 10. Spectral energy distribution of SHBL J012308.7+342049.

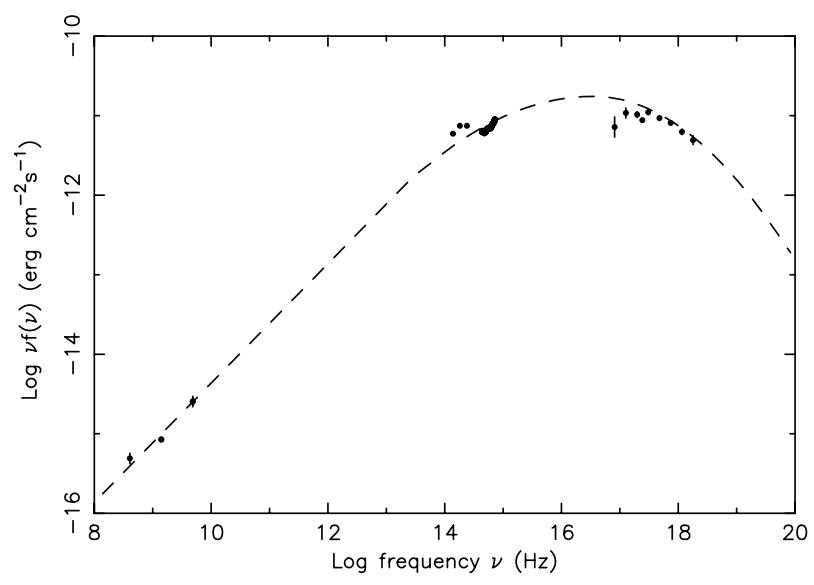

Fig. 11. Spectral energy distribution of SHBL J013632.5+390559.

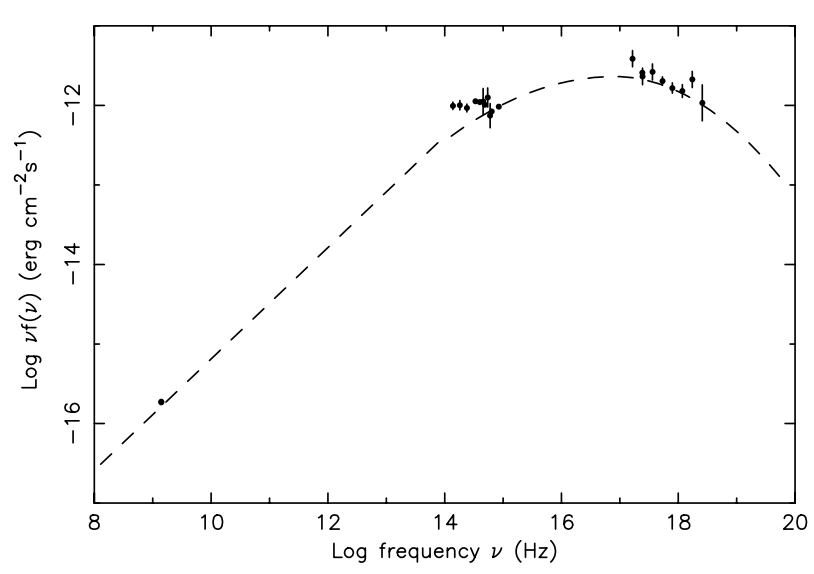

Fig. 12. Spectral energy distribution of SHBL J020106.3+003401.

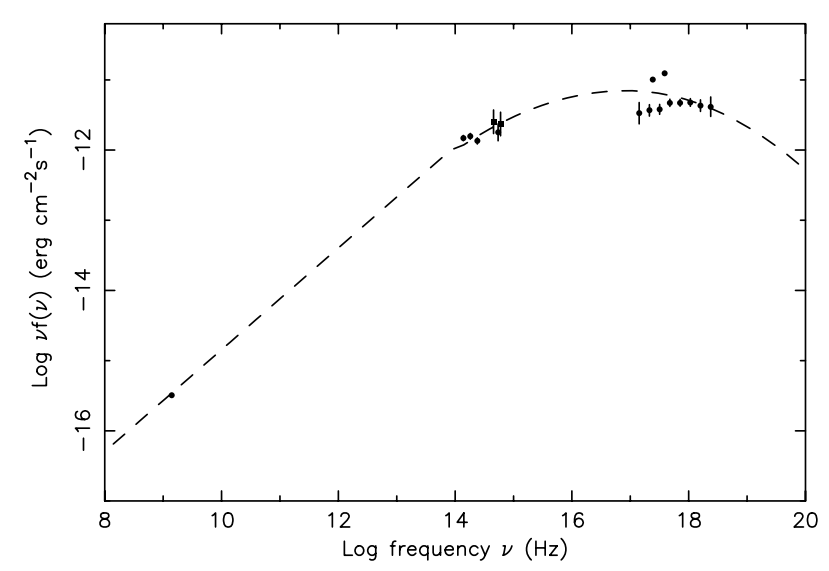

Fig. 13. Spectral energy distribution of SHBL J031951.9+184534.

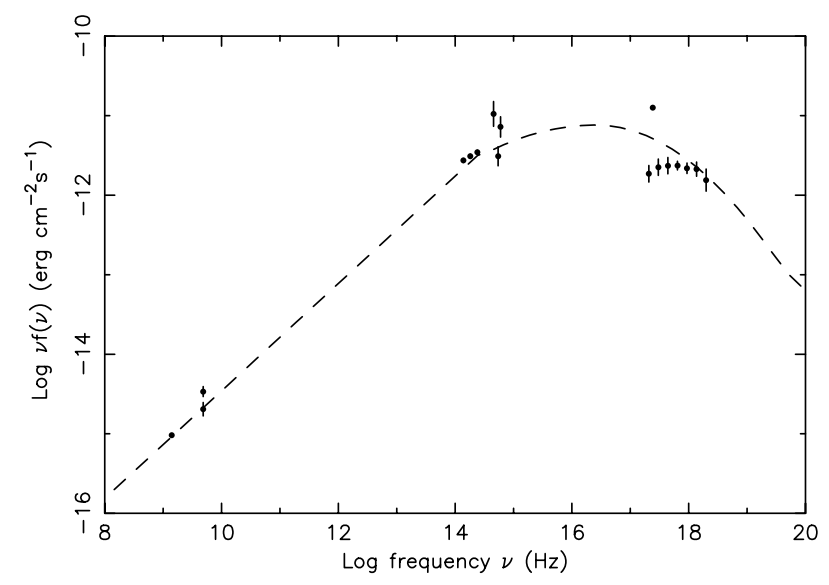

Fig. 14. Spectral energy distribution of SHBL J032613.9+022515.

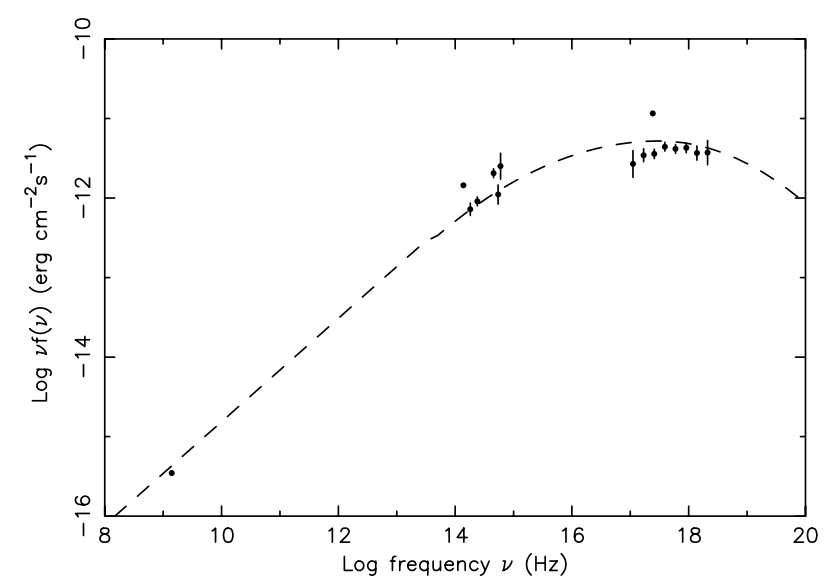

Fig. 15. Spectral energy distribution of SHBL J034923.2-115927. 
P. Giommi et al.: The sedentary survey of extreme high energy peaked BL Lacs. II., Online Material p 3

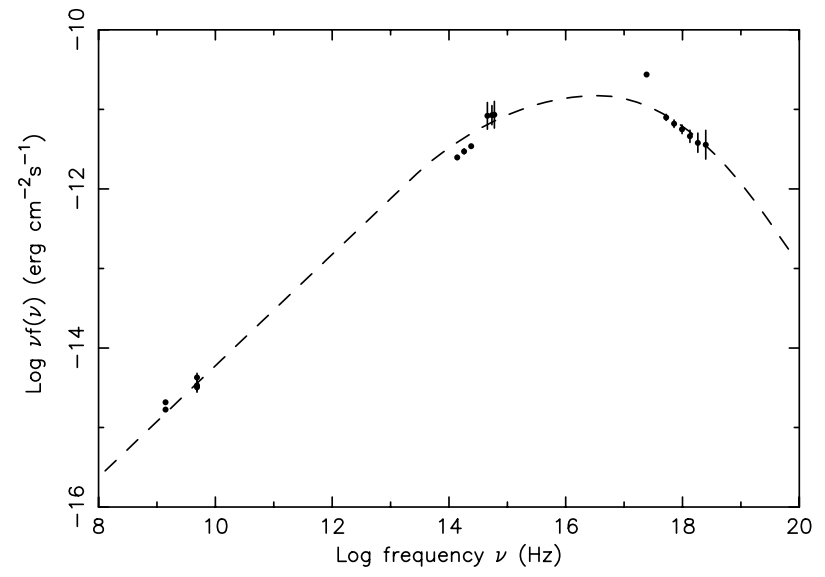

Fig. 16. Spectral energy distribution of SHBL J041652.4+010524.

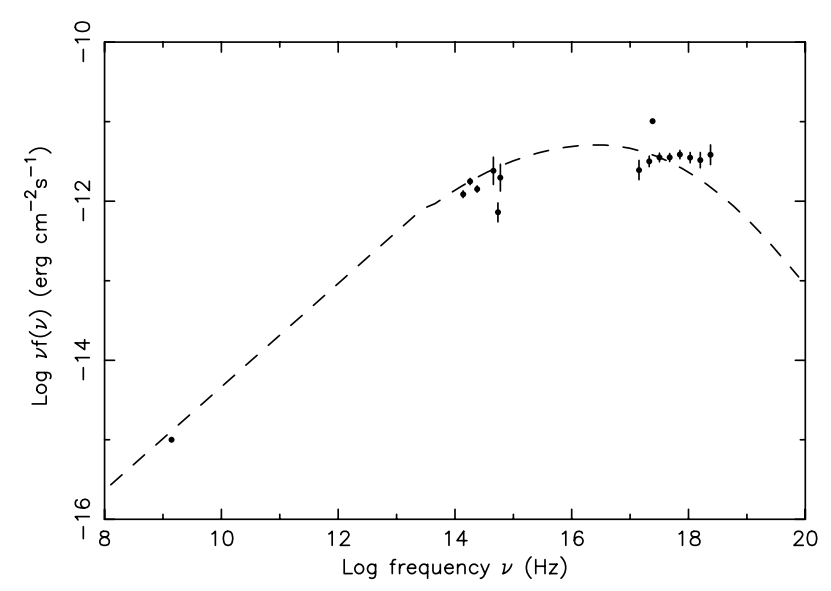

Fig. 17. Spectral energy distribution of SHBL J050939.0-040036.

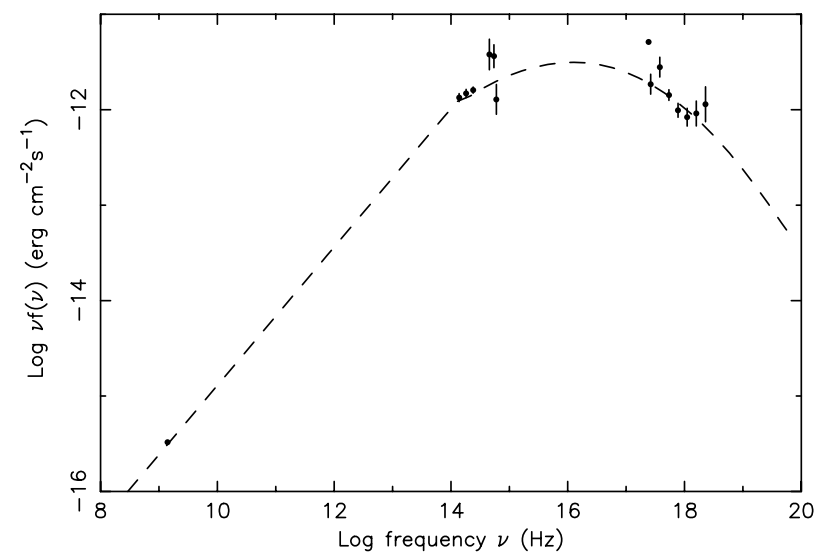

Fig. 18. Spectral energy distribution of SHBL J074405.6+743358.

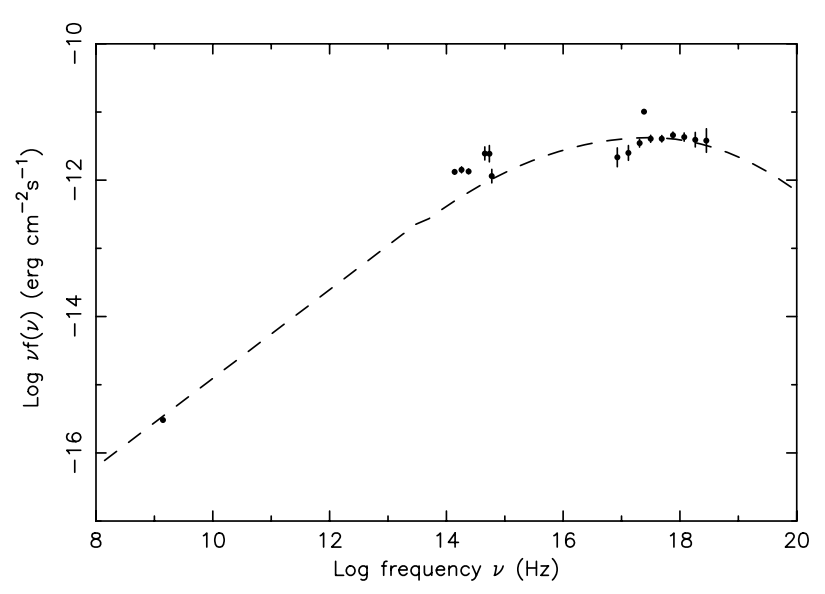

Fig. 19. Spectral energy distribution of SHBL J093037.5+495025.

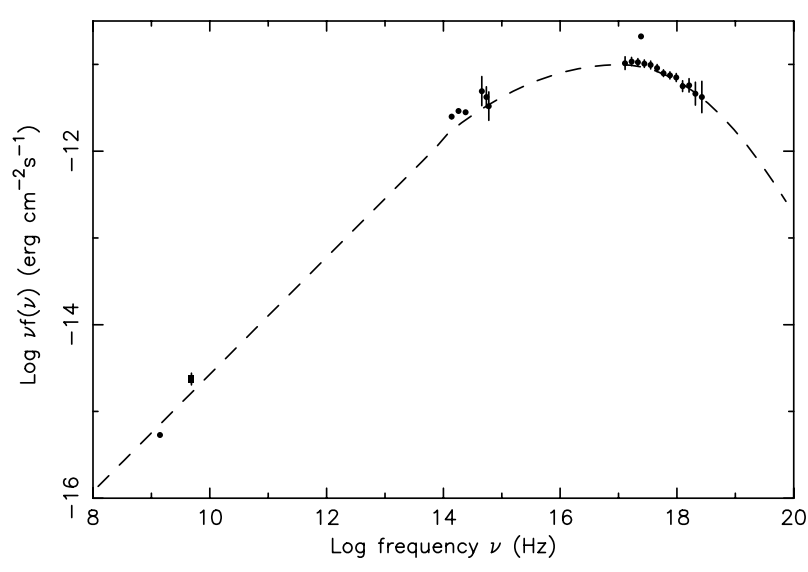

Fig. 20. Spectral energy distribution of SHBL J103118.6+505335.

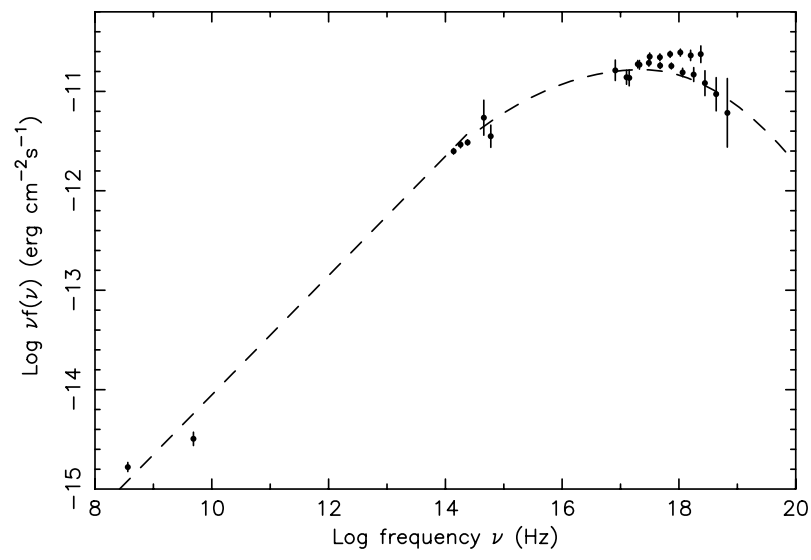

Fig. 21. Spectral energy distribution of SHBL J110337.6-232931. 
P. Giommi et al.: The sedentary survey of extreme high energy peaked BL Lacs. II., Online Material p 4

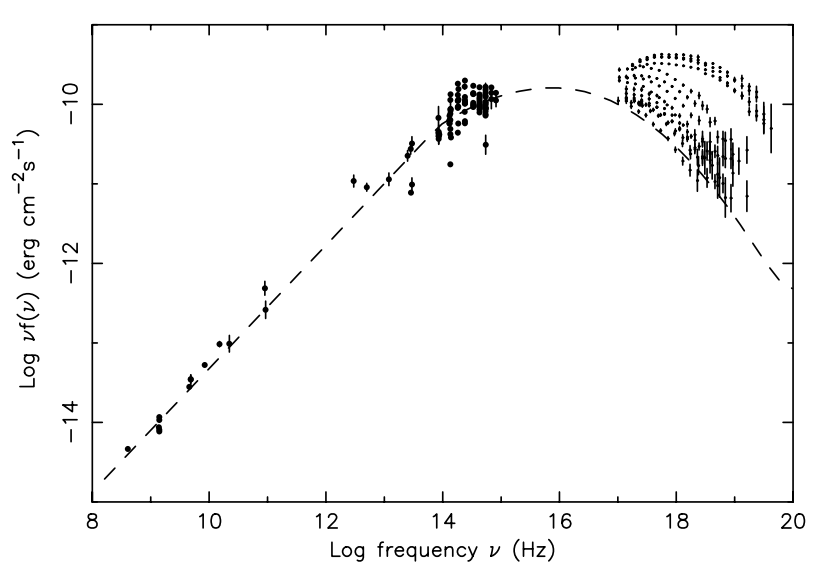

Fig. 22. Spectral energy distribution of SHBLJ $110427.3+381231$ $=$ MKN 421 .

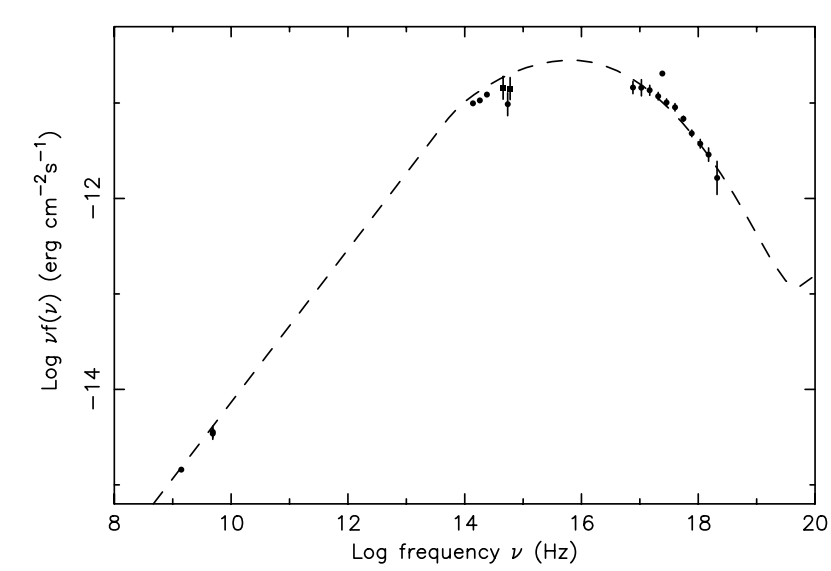

Fig. 23. Spectral energy distribution of SHBLJ 111706.3+201407.

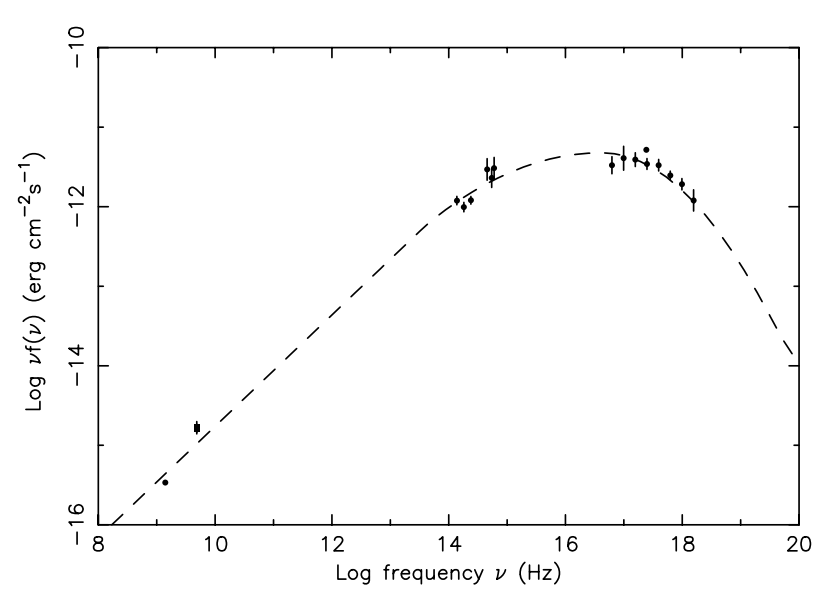

Fig. 24. Spectral energy distribution of SHBLJ 112048.0+421212.

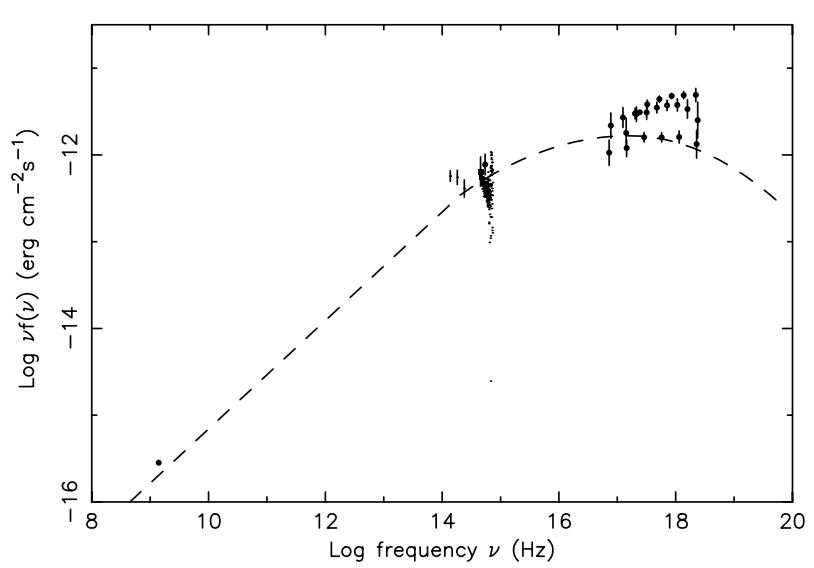

Fig. 25. Spectral energy distribution of SHBLJ 121158.6+224232.

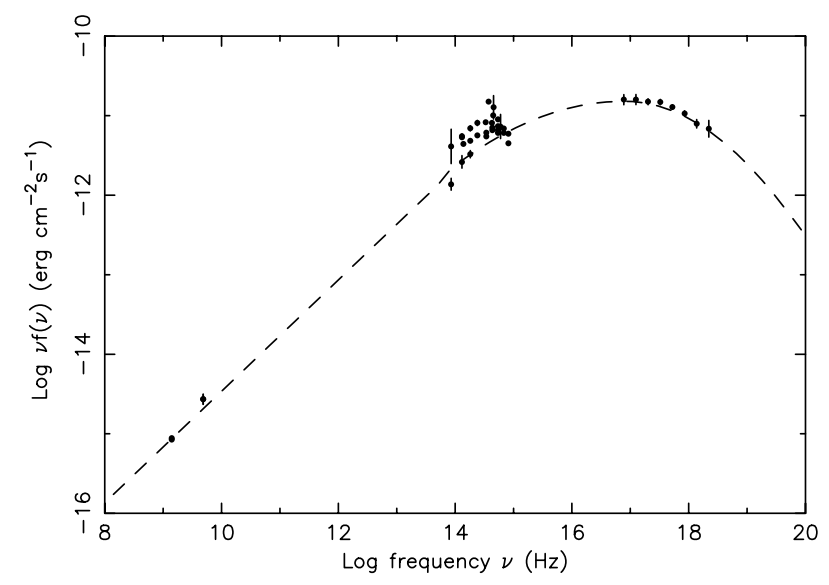

Fig. 26. Spectral energy distribution of SHBLJ 122121.9+301037.

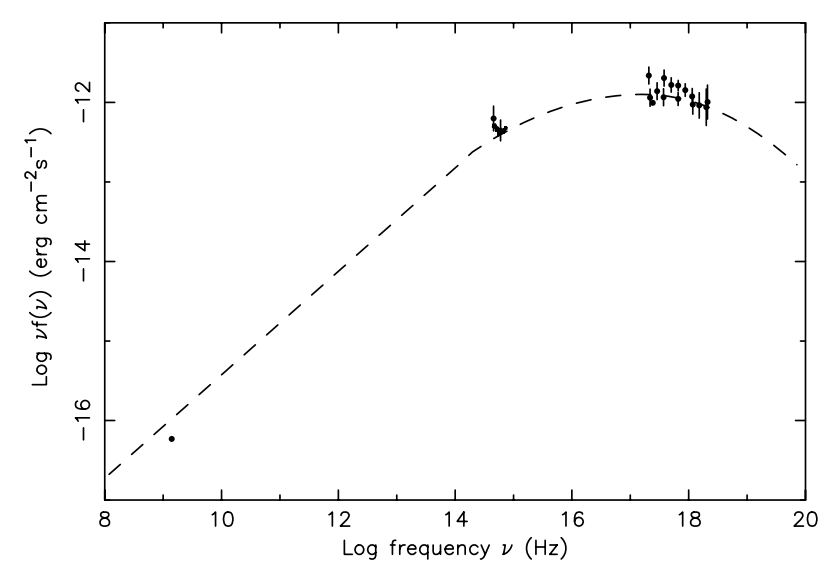

Fig. 27. Spectral energy distribution of SHBLJ 123511.0-140322. 
P. Giommi et al.: The sedentary survey of extreme high energy peaked BL Lacs. II., Online Material p 5

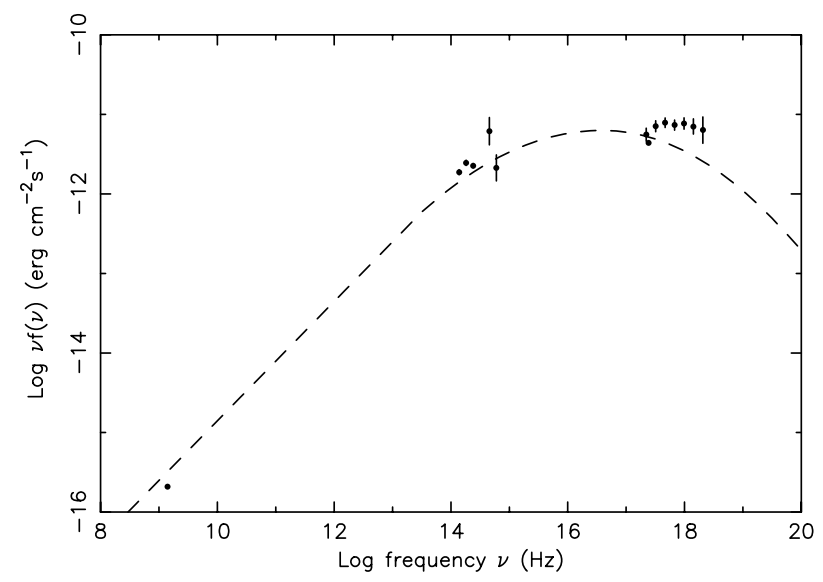

Fig. 28. Spectral energy distribution of SHBLJ 125731.9+241240.

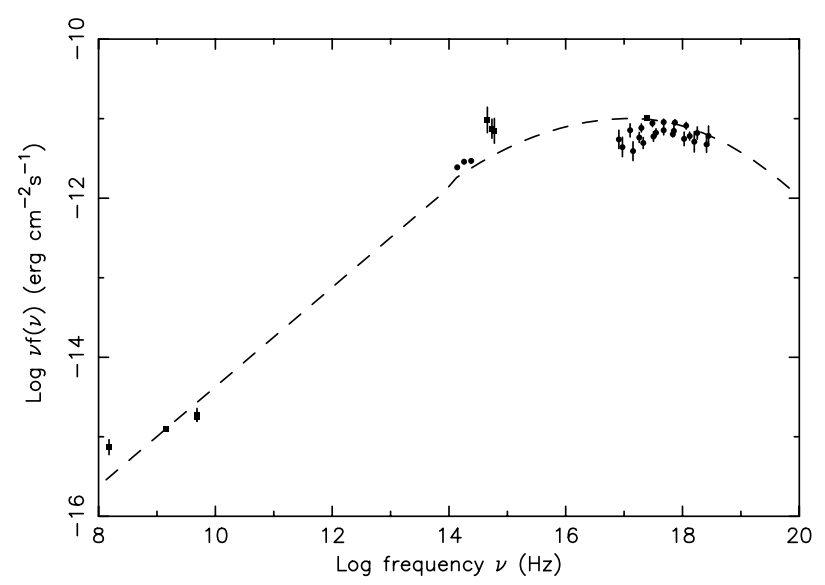

Fig. 29. Spectral energy distribution of SHBLJ $141756.1+254356$.

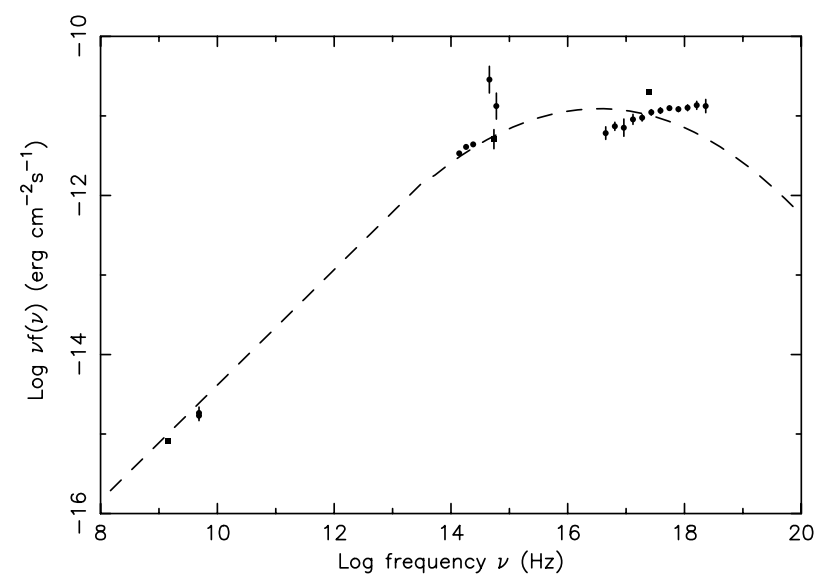

Fig. 30. Spectral energy distribution of SHBLJ 142832.6+424024.

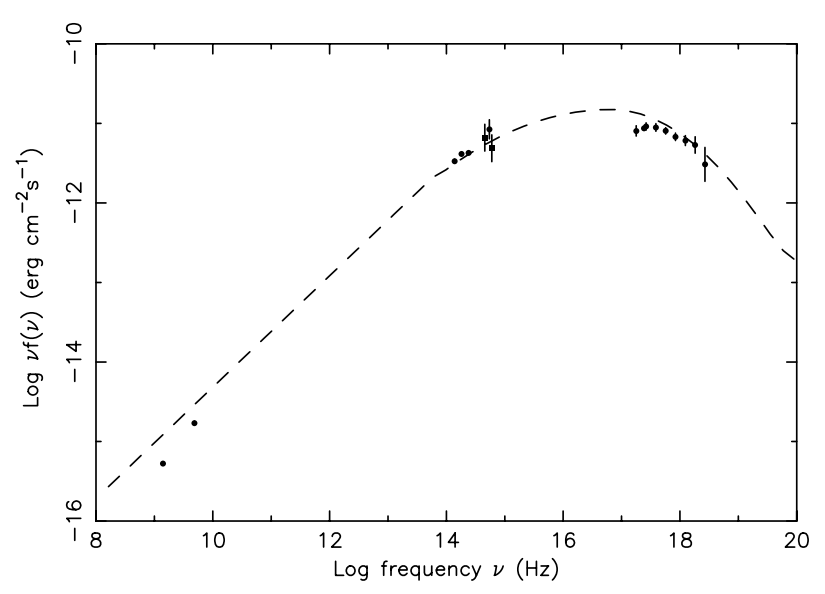

Fig. 31. Spectral energy distribution of SHBLJ 151747.4+652523.

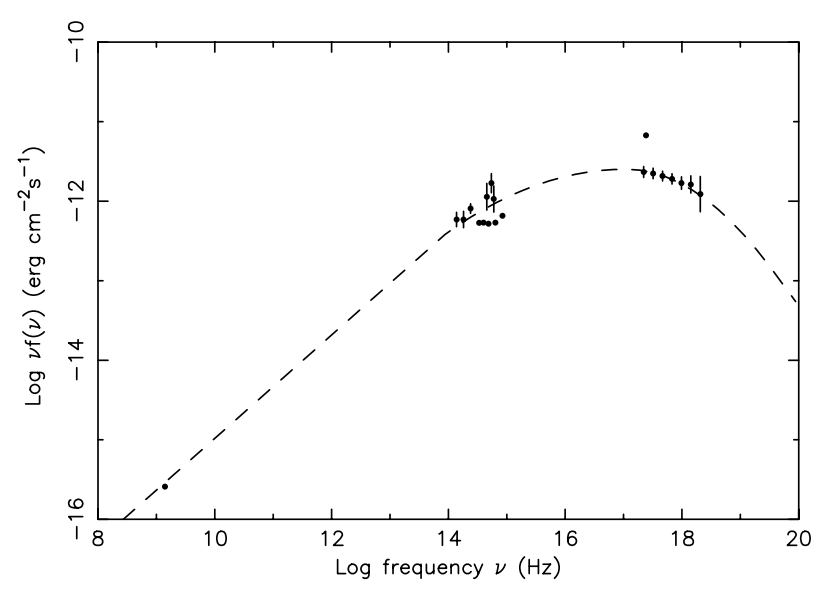

Fig. 32. Spectral energy distribution of SHBLJ 153500.9+532037.

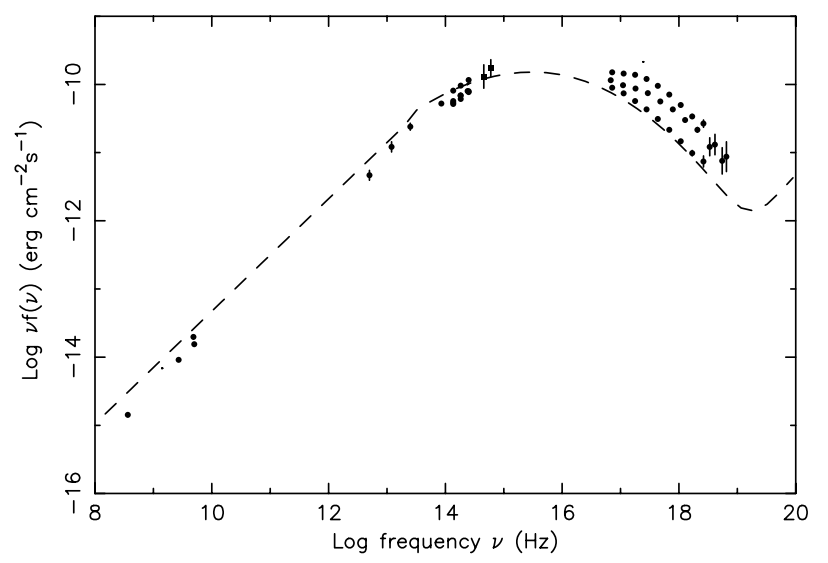

Fig. 33. Spectral energy distribution of SHBLJ 215852.0-301331 $=$ PKS 2155-304. 
P. Giommi et al.: The sedentary survey of extreme high energy peaked BL Lacs. II., Online Material p 6

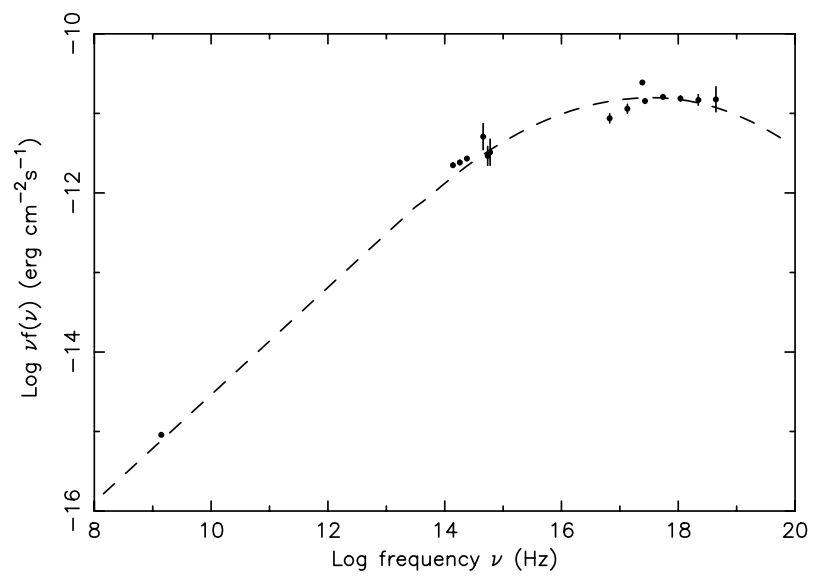

Fig. 34. Spectral energy distribution of SHBLJ 235907.9-303739. 